\title{
Drag associated with 3D trapped lee waves over an axisymmetric obstacle in two-layer atmospheres
}

Article

Accepted Version

Teixeira, M. A. C. and Miranda, P. M. A. (2017) Drag associated with 3D trapped lee waves over an axisymmetric obstacle in two-layer atmospheres. Quarterly Journal of the Royal Meteorological Society, 143 (709). pp. 3244-3258. ISSN 1477-870X doi: https://doi.org/10.1002/qj.3177 Available at https://centaur.reading.ac.uk/73133/

It is advisable to refer to the publisher's version if you intend to cite from the work. See Guidance on citing.

To link to this article DOI: http://dx.doi.org/10.1002/qj.3177

Publisher: Royal Meteorological Society

All outputs in CentAUR are protected by Intellectual Property Rights law, including copyright law. Copyright and IPR is retained by the creators or other copyright holders. Terms and conditions for use of this material are defined in the End User Agreement.

www.reading.ac.uk/centaur 
Central Archive at the University of Reading

Reading's research outputs online 
Drag associated with 3D trapped lee waves over an axisymmetric obstacle in two-layer atmospheres

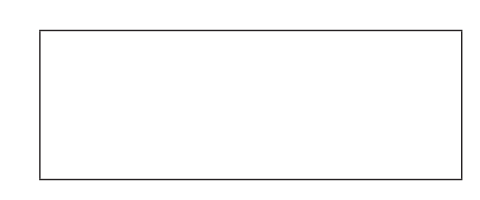

\author{
M. A. C. Teixeira ${ }^{a *}$ and P. M. A. Miranda ${ }^{b}$

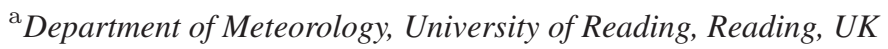 \\ ${ }^{\mathrm{b}}$ Instituto Dom Luiz, Faculdade de Ciências, University of Lisbon, Lisbon, Portugal
}

Mountain wave drag is evaluated explicitly using linear theory and verified against numerical simulations for the flow of idealized two-layer atmospheres with piecewiseconstant stratification over an axisymmetric mountain. Static stability is either higher in the bottom layer and lower in the top layer (Scorer's atmosphere), or neutral in the bottom layer and positive in the top layer, separated by a sharp temperature inversion (Vosper's atmosphere). The drag receives contributions from long mountain waves propagating vertically in the upper layer and from short trapped lee waves propagating downstream either in the lower layer, or at the inversion. This trapped lee wave drag, which is typically not represented in parametrizations, acts on the atmosphere at low levels. As in flow over a 2D ridge, this drag has several maxima as a function of the height of the interface between the two layers for Scorer's atmosphere, and is maximized by a marked Scorer parameter contrast between those layers. In Vosper's atmosphere, there is a single trapped lee wave drag maximum for Froude numbers near one, when the wind speed matches the phase speed of the dominant interfacial waves, and this drag is maximized for relatively low interface elevations, for which waves at the inversion have higher amplitude. The 3D flow geometry allows resonant wave modes to have various horizontal orientations and a continuous spectrum, forming a dispersive 'Kelvin ship wave' pattern, and expanding the regions in parameter space where the drag is non-zero relative to $2 \mathrm{D}$ flow, but it also dispersively decreases the drag magnitude. Nevertheless, the trapped lee wave drag on an axisymmetric obstacle can still equal or exceed the drag associated with vertically propagating waves and the reference hydrostatic drag valid for a uniformly stratified atmosphere. 
Key Words: Flow over orography; mountain wave drag; trapped lee waves; 3D flow; Non-hydrostatic effects; linear theory; numerical modelling

Received...

\section{Introduction}

Mountain wave drag is typically a subgrid-scale process in global weather and climate prediction models, therefore it must be parametrized (Stensrud,2009; Teixeira,2014). As the resolution of these models increases, non-hydrostatic waves progressively become more important among those that need to be represented in parametrizations, because non-hydrostatic effects are primarily felt at relatively short horizontal scales. The results of Steeneveld et al. (2008) and Tsiringakis et al. (2017) suggest that the wave drag associated with small-scale orography may be currently incorrectly represented in some models as turbulent form drag (which has a different dependence on the flow parameters), degrading their performance.

It is usually assumed that non-hydrostatic waves give a modest contribution to the globally-integrated drag compared to hydrostatic waves, because that is what can be inferred from linear theory for an atmosphere with constant wind and static stability (Gill,1982). For this reason, and also because non-hydrostatic waves propagate downstream of the orography that generates them, which implies that their impact on the atmosphere cannot be treated accurately using a single-column approach, these waves are usually neglected in parametrizations (Lott and Miller,1997), which use that kind of approach.

However, the former argument ignores the effect of the vertical structure of the atmosphere on the wave dynamics. A specific type of non-hydrostatic waves that are confined near the surface rather than propagating vertically are trapped lee waves (Scorer,1949). It has been shown recently that these waves can give a very substantial contribution to the drag in some parameter regimes, under specific atmospheric conditions conducive to resonance and constructive wave interference. This was illustrated by Teixeira et al. (2013a) for the atmospheric profile of Scorer (1949), where there is a stably stratified layer near the surface and a less stable layer aloft, and by Teixeira et al. (2013b) for the atmosphere of Vosper (2004), where the air is stably stratified aloft but near the surface has a neutral layer, capped by a sharp temperature inversion. Teixeira et al. (2013a, 2013b) calculated explicitly the drag produced by trapped lee waves for these idealized two-layer atmospheres, in flow over a 2D mountain ridge (the type of orography originally considered by both Scorer and Vosper). More recently, Sachsperger et al. (2015) studied the influence of stability in the upper stratified layer, for the second type of atmosphere, on the structural characteristics of waves at the inversion.

Flow over an elongated (approximately 2D) ridge is often considered the most relevant flow configuration for trapped lee waves, because it produces an extensive downstream wake of nearly monochromatic waves, which may be associated with rotors for waves of sufficient amplitude (Doyle and Durran,2007). However, from a parametrization viewpoint, the extension of these results to three dimensions is crucial, as the global orography is represented in a number of influential weather prediction models (for example, the ECMWF model and the UK Met Office model) as a set of isolated mountains with elliptical horizontal cross-sections (Phillips, 1984).

A good number of studies have addressed 3D trapped lee waves using linear theory, e.g.: Scorer (1956), Scorer and Wilkinson (1956), Crapper (1959), Sawyer (1962), Marthinsen (1980) and Simard and Peltier (1982). Some other studies used numerical simulations, for example Peltier and Clark (1983), and more recently Broutman et al. (2003), but none of these studies focused specifically on the drag associated with these waves. An exception to this is the study of Héreil and Stein (1999), which presents a particular case of drag produced by a trapped lee wave in an stratified atmosphere where the wind increases with height (the same setting as adopted by Sharman and Wurtele $(1983,2004))$. Some work has also been done on the related, but simpler, problem of lee waves trapped at a density interface (which are essentially similar to ship waves), both using numerical simulations (Jiang and Smith, 2000 ; Esler et al. 2007), and laboratory experiments (Lacaze et al.,2013). Although in Jiang and Smith (2000) and Esler et al. (2007) a drag coefficient 
was evaluated, the focus of these studies was primarily on the flow structure. In the very recent study of Teixeira et al. (2017), an explicit formula for the drag produced by 3D trapped lee waves of the simplest possible type (waves propagating at the interface between two constant-density fluids) was calculated theoretically, and compared with the drag measured in laboratory experiments. This can be considered a direct precursor of the present study, where the theoretical analysis, and the numerical simulations used to test it, are extended to the more meteorologically relevant static stability profiles adopted by Teixeira et al. (2013a, 2013b).

A general expression for the momentum flux associated with 3D trapped lee waves (which equals the surface drag in the absence of critical levels) was presented in the pioneering analytical study of Bretherton (1969) as a generalization of the corresponding 2D expression. However, Bretherton considered the situation of a flow bounded above by a rigid lid, which is not very relevant to the atmosphere. Bretherton's 2D trapped lee wave drag expression was extended to a vertically unbounded atmosphere by Smith (1976), but it was only Gregory et al. (1998), in the context of the drag parametrization adopted in the UK Met Office's Unified Model, that extended the 3D trapped lee wave expression of Bretherton to an unbounded atmosphere (their equation (22)). Although implemented for some time, this component of the drag was subsequently removed from the parametrization. As Smith's (1976) corresponding 2D expression, the 3D trapped lee wave drag expression of Gregory et al. (1998) is too general for studying the behaviour of the drag with the incoming flow parameters in a systematic way. That will be done in the present study for the atmospheres of Scorer (1949) and Vosper (2004).

Towards that aim, the calculations of Teixeira et al. (2013a,2013b) for 2D trapped lee waves are extended here to the simplest possible case of 3D orography: an axisymmetric mountain. Unlike in the 2D case, where the spectrum of trapped lee waves contributing to the drag is discrete, in the 3D case this spectrum, as the spectrum of vertically propagating waves, is continuous, corresponding to a "shipwave" pattern originating above the obstacle instead of a train of monochromatic trapped lee waves. Consequently, the form taken by the drag component due to trapped lee waves is no longer a closed analytical expression, but rather a 1D integral in wavenumber space (as found also by Teixeira et al. (2017)).

The remainder of this paper is organized as follows: Section 2 contains a description of the trapped lee wave drag model proposed here. In section 3 the model is tested for the two idealized atmospheres considered, for representative input parameters, and verified against numerical simulations. Finally, in Section 4, some concluding remarks are presented.

\section{Theoretical model}

As in Teixeira et al. (2013a, 2013b), the point of departure is the Taylor-Goldstein equation (but now for 3D flow), where it is assumed that the Boussinesq approximation is valid, the flow is steady, inviscid, adiabatic, non-rotating and linearized with respect to a background incoming state:

$$
\hat{w}^{\prime \prime}+\left[\frac{N^{2} \kappa^{2}}{(U k+V l)^{2}}-\frac{U^{\prime \prime} k+V^{\prime \prime} l}{U k+V l}-\kappa^{2}\right] \hat{w}=0
$$

Here $\hat{w}$ is the Fourier transform of the vertical velocity perturbation associated with the waves, the primes denote differentiation with respect to height $z, N=\left[\left(g / \theta_{0}\right)(d \bar{\theta} / d z)\right]^{1 / 2}$ is the Brunt Väisälä frequency (where $\bar{\theta}(z)$ is the potential temperature of the background flow and $\theta_{0}$ is its reference value - assumed to be constant), $(U, V)$ is the background wind velocity, $(k, l)$ is the horizontal wavenumber vector and $\kappa=\left(k^{2}+l^{2}\right)^{1 / 2}$ is its magnitude.

The wind and static stability profiles of the incoming flow are similar to those used by Teixeira et al. (2013a, 2013b), namely the wind velocity is constant and (without loss of generality) assumed to be in the $x$ direction. Then, the second term within square brackets in (1), involving the wind profile curvature, vanishes. Defining the Scorer parameter as $l_{S}=N / U$, (1) reduces to

$$
\hat{w}^{\prime \prime}+\frac{\kappa^{2}}{k^{2}}\left(l_{S}^{2}-k^{2}\right) \hat{w}=0 .
$$


(a)

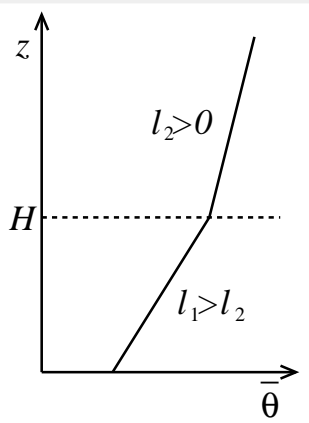

(b)

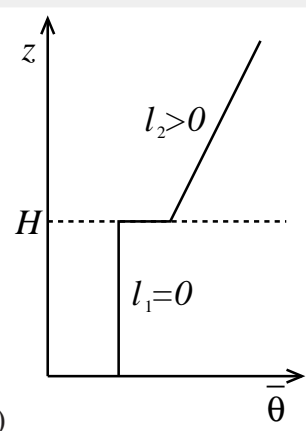

Figure 1. Schematic diagram of the background potential temperature profiles used here as a function of height. $l_{1}$ and $l_{2}$ are the values of the Scorer parameter $l_{S}$ in the lower and upper layers, respectively. $H$ is the height of the interface between the two layers. (a) Profile of Scorer (1949) or Teixeira et al. (2013a), where $l_{S}$ is piecewise constant, and larger in the lower layer. (b) Profile of Vosper (2004) or Teixeira et al. (2013b), where $l_{S}$ is also piecewise constant, but zero in the lower layer and positive in upper one, with a sharp temperature inversion in between.

Concerning the static stability, two different two-layer profiles for the Brunt-Väisälä frequency, or equivalently for the Scorer parameter, will be considered. In the first one, following Scorer (1949) and Teixeira et al. (2013a), the Scorer parameter in a lower layer near the surface is higher than that in an upper semi-infinite layer, i.e.

$$
l_{S}= \begin{cases}l_{2}>0 & \text { if } \quad z>H \\ l_{1}>l_{2} & \text { if } \quad 0<z \leq H\end{cases}
$$

whe where $l_{1}$ and $l_{2}$ are constant, and the top of the lower layer is at $z=H$. The second profile, which follows Vosper (2004) and Teixeira et al. (2013b), has a neutral layer near the surface, topped by a sharp temperature inversion, followed by a statically stable layer above, i.e.

$$
l_{S}=\left\{\begin{array}{lll}
l_{2}>0 & \text { if } & z>H \\
+\infty & \text { if } & z=H \\
l_{1}=0 & \text { if } & 0<z<H .
\end{array}\right.
$$
condition at the surface,

$$
\hat{w}_{1}(z=0)=i U k \hat{h},
$$

where $i=\sqrt{-1}$, and $\hat{h}$ is the Fourier transform of the ground elevation function $h(x, y)$, specifying that the flow is tangent to the orography at the surface. Here it will be assumed, for illustrative purposes, that the orography is a bell-shaped axisymmetric mountain given by

$$
h(x, y)=\frac{h_{0}}{\left[1+(x / a)^{2}+(y / a)^{2}\right]^{3 / 2}} \Rightarrow \hat{h}(k, l)=\frac{1}{2 \pi} h_{0} a^{2} e^{-a \kappa},
$$

where $a$ and $h_{0}$ are its half-width and maximum height, but the results would not change qualitatively for other isolated axisymmetric orographies. Additionally, both $\hat{w}$ and the Fourier transform of the pressure perturbation associated with the waves must be continuous at the interface between the two layers. In the first case (since there is no mean wind shear), this amounts to imposing $\hat{w}_{1}(H)=\hat{w}_{2}(H)$ and $\hat{w}_{1}^{\prime}(z=H)=\hat{w}_{2}^{\prime}(z=H)$, but in the second case, since there is a discontinuity in the potential temperature, the boundary-condition 
on the pressure is expressed as

$$
\hat{w}_{1}^{\prime}(z=H)-\hat{w}_{2}^{\prime}(z=H)=\frac{g^{\prime}}{U^{2}} \frac{\kappa^{2}}{k^{2}} \hat{w}_{1}(z=H),
$$

where $g^{\prime}=g \Delta \theta / \theta_{0}$ and $\Delta \theta$ are the reduced gravity and the potential temperature jump at the inversion, respectively. Note that (7) is an extension to 3D of the corresponding boundary condition formulated by Vosper (2004) (his Eq. (9)). It can also be understood as a non-rotating and stationary version of the boundary condition implemented by Eq. (11) of McPhee and Kantha (1989). One possible way of obtaining (7) is by integrating (1) across the inversion, while taking the limits immediately above and below $z=H$. Finally, the upper boundary condition in the upper layer is either a radiation boundary-condition, for vertically propagating waves, or a condition expressing exponential decay as $z \rightarrow+\infty$, for evanescent waves.

The drag is caused the pressure perturbation associated with the waves at the surface. The Fourier transform of this pressure perturbation is given by (cf. Eq. (17) of Teixeira, 2014)

$$
\hat{p}(z=0)=-i \frac{\rho_{0} k}{\kappa^{2}} U \hat{w}_{1}^{\prime}(z=0)
$$

where $\rho_{0}$ is a reference density (assumed to be constant). This equation can be obtained by taking the Fourier transform of the equation for the horizontal divergence of the wave velocity perturbation, and expressing that divergence in terms of $\hat{w}^{\prime}$ from mass conservation. The drag exerted by the waves on the orography may be expressed as (Teixeira et al. 2017)

$$
D=4 \pi^{2} i \int_{-\infty}^{+\infty} \int_{-\infty}^{+\infty} k \hat{p}^{*}(z=0) \hat{h} d k d l=8 \pi^{2} \operatorname{Im} \int_{-\infty}^{+\infty} \int_{0}^{+\infty} k \hat{p}(z=0) \hat{h}^{*} d k d l
$$

where Im denotes “imaginary part”, and the asterisk denotes complex conjugate. The first expression makes use of Parseval's theorem, and the second one of the fact that drag is a real quantity. Note that, because the mountains to be considered are axisymmetric, this drag points in the $x$ direction, the direction of the incoming wind velocity.

\subsection{Atmosphere of Scorer (1949)}

For the profile of the Scorer parameter (3), illustrated in Figure 1(a), the solutions to (2) in the lower layer $(z<H)$ take the form:

$$
\hat{w}_{1}=\left\{\begin{array}{lll}
a_{1} e^{i m_{1} z}+b_{1} e^{-i m_{1} z} & \text { if } & |k|<l_{1} \\
c_{1} e^{-n_{1} z}+d_{1} e^{n_{1} z} & \text { if } & |k|>l_{1}
\end{array}\right.
$$

where $m_{1}=\frac{\kappa}{k}\left(l_{1}^{2}-k^{2}\right)^{1 / 2}$ and $n_{1}=\frac{\kappa}{|k|}\left(k^{2}-l_{1}^{2}\right)^{1 / 2}$, and $a_{1}, b_{1}, c_{1}$ e $d_{1}$ are coefficients to be determined. The first solution corresponds to waves that propagate vertically, whereas the second one corresponds to waves that are evanescent. In the upper layer the solutions are

$$
\hat{w}_{2}=\left\{\begin{array}{lll}
a_{2} e^{i m_{2} z} & \text { if } & |k|<l_{2} \\
c_{2} e^{-n_{2} z} & \text { if } & |k|>l_{2}
\end{array}\right.
$$

where $m_{2}=\frac{\kappa}{k}\left(l_{2}^{2}-k^{2}\right)^{1 / 2}$ and $n_{2}=\frac{\kappa}{|k|}\left(k^{2}-l_{2}^{2}\right)^{1 / 2}$, and $a_{2}$ and $c_{2}$ are unknown coefficients. Note that these solutions already incorporate the radiation or decay upper boundary conditions, respectively.

When the remaining boundary conditions are applied, all unknown coefficients may be determined. For the purpose of calculating the drag, it is enough to present $a_{1}$ and $b_{1}$, as the drag produced by the solution that is already evanescent in the lower layer (second 
line of (10)), for $|k|>l_{1}$, is zero (cf. Teixeira et al., 2013a), and $a_{2}$ and $c_{2}$ play no role in the surface pressure. Hence,

$$
a_{1}=\frac{i U k \hat{h}\left(m_{1}+m_{2}\right) e^{-i m_{1} H}}{2 m_{1} \cos \left(m_{1} H\right)-2 i m_{2} \sin \left(m_{1} H\right)}, \quad b_{1}=\frac{i U k \hat{h}\left(m_{1}-m_{2}\right) e^{i m_{1} H}}{2 m_{1} \cos \left(m_{1} H\right)-2 i m_{2} \sin \left(m_{1} H\right)},
$$

if $|k|<l_{2}$ or

$$
a_{1}=\frac{U k \hat{h}\left(i m_{1}-n_{2}\right) e^{-i m_{1} H}}{2 m_{1} \cos \left(m_{1} H\right)+2 n_{2} \sin \left(m_{1} H\right)}, \quad b_{1}=\frac{U k \hat{h}\left(i m_{1}+n_{2}\right) e^{i m_{1} H}}{2 m_{1} \cos \left(m_{1} H\right)+2 n_{2} \sin \left(m_{1} H\right)},
$$

if $l_{2}<|k|<l_{1}$.

From (8) and (10), the Fourier transform of the pressure perturbation may be written in terms of $a_{1}$ and $b_{1}$ as

$$
\hat{p}(z=0)=\frac{\rho_{0} k}{\kappa^{2}} U m_{1}\left(a_{1}-b_{1}\right),
$$

where (12) or (13) may be used, depending on the range of wavenumbers considered. The drag (9) may be split into two terms, according to the range of wavenumbers that contribute to it: $D=D_{I}+D_{L}$, where

$$
D_{I}=8 \pi^{2} \operatorname{Im} \int_{-\infty}^{+\infty} \int_{0}^{l_{2}} k \hat{p}(z=0) \hat{h}^{*} d k d l, \quad D_{L}=8 \pi^{2} \operatorname{Im} \int_{-\infty}^{+\infty} \int_{l_{2}}^{l_{1}} k \hat{p}(z=0) \hat{h}^{*} d k d l
$$

are henceforth referred to as "internal wave drag" and "trapped lee wave drag", respectively. Using (12), (13), (14) and (15), $D_{I}$ and $D_{L}$ may be calculated explicitly, yielding

$$
D_{I}=8 \pi^{2} \rho_{0} U^{2} \int_{-\infty}^{+\infty} \int_{0}^{l_{2}} \frac{k^{3} m_{1}^{2} m_{2}|\hat{h}|^{2}}{\kappa^{2}\left[m_{1}^{2} \cos ^{2}\left(m_{1} H\right)+m_{2}^{2} \sin ^{2}\left(m_{1} H\right)\right]} d k d l
$$

and

$$
D_{L}=8 \pi^{2} \rho_{0} U^{2} \operatorname{Im} \int_{-\infty}^{+\infty} \int_{l_{2}}^{l_{1}} \frac{k^{3} m_{1}|\hat{h}|^{2}}{\kappa^{2}} \frac{m_{1} \sin \left(m_{1} H\right)-n_{2} \cos \left(m_{1} H\right)}{m_{1} \cos \left(m_{1} H\right)+n_{2} \sin \left(m_{1} H\right)} d k d l
$$

Equation (16) was obtained by taking the imaginary part of the integrand, since this has no singularities. In (17), on the other hand, since the integrand is real, the imaginary part of the integral may only come from singularities. These occur when the denominator of the integrand is zero, namely when

$$
\tan \left(m_{1} H\right)=-\frac{m_{1}}{n_{2}}
$$

as originally derived by Scorer (1949), with the difference that $m_{1}$ and $n_{2}$ have a more complicated definition for 3D flow. Complex integration may be used to simplify the inner integral in (17), as shown by Teixeira et al. (2013a) for the equivalent 2D flow, and more recently for an analogous 2D integral (describing a 3D flow) by Teixeira et al. (2017). The procedure for the complex integration in the latter case is detailed in the Appendix of Teixeira et al. (2017), following roughly the same approach as in Appendix B of Teixeira et al. (2013a), but without using a change of variable.

The two drag components $D_{I}$ and $D_{L}$ may be made dimensionless by dividing them by the hydrostatic drag that would be produced if the lower layer with Scorer parameter $l_{1}$ extended up to infinity. This is (cf. Eq. (26) of Teixeira, 2014)

$$
D_{0}=\frac{\pi}{4} \rho_{0} U^{2} l_{1} a h_{0}^{2} .
$$


Applying this normalization, (16) becomes

$$
\frac{D_{I}}{D_{0}}=64 \pi \frac{1}{l_{1}^{\prime}}\left(\frac{a}{H}\right)^{3} \int_{0}^{+\infty} \int_{0}^{l_{2}^{\prime}} \frac{\left.k^{\prime 3} m_{1}^{\prime 2} m_{2}^{\prime} \hat{h}^{\prime}\right|^{2}}{\kappa^{\prime 2}\left[m_{1}^{\prime 2} \cos ^{2}\left(m_{1}^{\prime}\right)+m_{2}^{\prime 2} \sin ^{2}\left(m_{1}^{\prime}\right)\right]} d k^{\prime} d l^{\prime},
$$

where all primed variables except $\hat{h}^{\prime}=\hat{h} /\left(h_{0} a^{2}\right)$ are normalized by being multiplied by $H$. Similarly, after (17) is simplified using contour integration and normalized, it becomes

$$
\frac{D_{L}}{D_{0}}=64 \pi^{2} \frac{1}{l_{1}^{\prime}}\left(\frac{a}{H}\right)^{3} \sum_{j} \int_{0}^{+\infty} \frac{k_{j}^{\prime 4} m_{1}^{\prime 2}\left(k_{j}^{\prime}\right) n_{2}^{\prime}\left(k_{j}^{\prime}\right)\left|\hat{h}^{\prime}\right|^{2}\left(k_{j}^{\prime}\right)}{\kappa_{j}^{\prime 4}+n_{2}^{\prime}\left(k_{j}^{\prime}\right)\left[m_{1}^{\prime 2}\left(k_{j}^{\prime}\right) l^{\prime 2}+\kappa_{j}^{\prime 4}\right]} d l^{\prime}
$$

where the index $j$ in the sum refers to the number of the trapped lee wave mode. $k_{j}^{\prime}$ is a resonant $k^{\prime}$ determined by (18), which may only be evaluated numerically, and $\kappa_{j}^{\prime}=\left(k_{j}^{\prime 2}+l^{\prime 2}\right)^{1 / 2}$. In (20) and (21) the coefficient 64 comes partly from the normalizing factor (19) and partly from symmetry of the integrands with respect to $l^{\prime}$.

Equations (20) and (21) should be compared with the corresponding expressions for 2D flow (Eqs. (29) and (30) of Teixeira et al. (2013a) with $U_{1}=U_{2}=U$ ). The main difference in (20)-(21) (apart from geometrical factors associated with 3D flow) is that (20) is given by a double integral instead of a single one, and (21) still contains an integral over $l^{\prime}$ instead of none. What this means is that the trapped lee wave modes are no longer discrete, but rather have a continuous wavenumber distribution. This has important consequences for the wave pattern (in essentially the same way as for pure interfacial waves in Teixeira et al. (2017)), as will be seen later.

The vertical velocity associated with the waves is given by

$$
w(x, y, z)=\int_{-\infty}^{+\infty} \int_{-\infty}^{+\infty} \hat{w}(k, l, z) e^{i(k x+l y)} d k d l
$$

In the lower layer, taking into account the symmetry of $a_{1}$ and $b_{1}$ with respect to $l$, (22) may be expressed using (10) as

$$
w_{1}(x, y, z)=2 \operatorname{Re} \int_{0}^{+\infty} \int_{0}^{+\infty}\left\{a_{1}(k, l)\left[e^{i\left(k x+l y+m_{1} z\right)}+e^{i\left(k x-l y+m_{1} z\right)}\right]+b_{1}(k, l)\left[e^{i\left(k x+l y-m_{1} z\right)}+e^{i\left(k x-l y-m_{1} z\right)}\right]\right\} d k d l
$$

The two terms within square brackets consist of waves making symmetric angles with the $x$ axis. As will be seen, this is associated with a triangular wake (akin to those investigated by Sharman and Wurtele (1983, 2004)), which comes mainly from the trapped lee wave field associated with wavenumbers $|k|$ in a range between $l_{2}$ and $l_{1}$. For these waves, it is possible to perform analogous complex integrations as used for the drag (essentially following Sawyer (1962), section 4) to calculate the resonant trapped lee wave modes that satisfy (18), yielding, after normalization

$$
\begin{aligned}
\frac{w_{L}(x, y, z)}{U\left(\frac{h_{0}}{a}\right)}= & 2 \pi\left(\frac{a}{H}\right)^{3} \sum_{j} \int_{0}^{+\infty} \frac{k_{j}^{\prime 2} m_{1}^{\prime}\left(k_{j}^{\prime}\right) \kappa_{j}^{\prime 2} n_{2}^{\prime}\left(k_{j}^{\prime}\right) \hat{h}^{\prime}\left(k_{j}^{\prime}\right)}{\kappa_{j}^{\prime 4}+n_{2}^{\prime}\left(k_{j}^{\prime}\right)\left[m_{1}^{\prime 2}\left(k_{j}^{\prime}\right) l^{\prime 2}+\kappa_{j}^{\prime 4}\right]}\left\{\sin \left[k_{j}^{\prime} x^{\prime}+l^{\prime} y^{\prime}-m_{1}^{\prime}\left(k_{j}^{\prime}\right) z^{\prime}\right]+\sin \left[k_{j}^{\prime} x^{\prime}-l^{\prime} y^{\prime}-m_{1}^{\prime}\left(k_{j}^{\prime}\right) z^{\prime}\right]\right. \\
& \left.-\sin \left[k_{j}^{\prime} x^{\prime}+l^{\prime} y^{\prime}+m_{1}^{\prime}\left(k_{j}^{\prime}\right) z^{\prime}\right]-\sin \left[k_{j}^{\prime} x^{\prime}-l^{\prime} y^{\prime}+m_{1}^{\prime}\left(k_{j}^{\prime}\right) z^{\prime}\right]\right\} d l^{\prime},
\end{aligned}
$$

where $w_{L}$ is the vertical velocity only associated with resonant trapped lee waves, $x^{\prime}=x / H, y^{\prime}=y / H$ and $z^{\prime}=z / H$. As in the original, simpler, calculation of Scorer (1949) for 2D trapped lee waves, this solution requires the addition of a term that cancels out the wave field for $x^{\prime}<0$ and doubles its amplitude for $x^{\prime}>0$. This can be viewed as implementing a radiation boundary-condition in the horizontal direction. Note that (24) does not satisfy the lower boundary-condition (5), and so is only formally valid far downstream of the obstacle. 
and

$$
\hat{w}_{1}=c_{1} e^{-\kappa z}+d_{1} e^{\kappa z},
$$

where $c_{1}$ and $d_{1}$ are coefficients to be determined. The waves are always evanescent in this layer because of the neutral stratification. In the upper layer the solutions are

$$
\hat{w}_{2}=\left\{\begin{array}{lll}
a_{2} e^{i m_{2} z} & \text { if } & |k|<l_{2} \\
c_{2} e^{-n_{2} z} & \text { if } & |k|>l_{2}
\end{array}\right.
$$

where $m_{2}$ and $n_{2}$ were defined previously and $a_{2}$ and $c_{2}$ are unknown coefficients. Again, (26) already incorporates the radiation or decay upper boundary condition.

When the remaining boundary conditions are applied, all unknown coefficients may be determined. For the purpose of calculating the drag, it is enough to present here $c_{1}$ and $d_{1}$, as $a_{2}$ and $c_{2}$ play no role in the surface pressure. Hence,

$$
c_{1}=\frac{1}{2} \frac{i U k \hat{h} e^{\kappa H}\left(\kappa-i m_{2}-\frac{g^{\prime}}{U^{2}} \frac{\kappa^{2}}{k^{2}}\right)}{\kappa \cosh (\kappa H)-\left(i m_{2}+\frac{g^{\prime}}{U^{2}} \frac{\kappa^{2}}{k^{2}}\right) \sinh (\kappa H)}, \quad d_{1}=\frac{1}{2} \frac{i U k \hat{h} e^{-\kappa H}\left(\kappa+i m_{2}+\frac{g^{\prime}}{U^{2}} \frac{\kappa^{2}}{k^{2}}\right)}{\kappa \cosh (\kappa H)-\left(i m_{2}+\frac{g^{\prime}}{U^{2}} \frac{\kappa^{2}}{k^{2}}\right) \sinh (\kappa H)},
$$

if $|k|<l_{2}$ or

$$
c_{1}=\frac{1}{2} \frac{i U k \hat{h} e^{\kappa H}\left(\kappa+n_{2}-\frac{g^{\prime}}{U^{2}} \frac{\kappa^{2}}{k^{2}}\right)}{\kappa \cosh (\kappa H)+\left(n_{2}-\frac{g^{\prime}}{U^{2}} \frac{\kappa^{2}}{k^{2}}\right) \sinh (\kappa H)}, \quad d_{1}=\frac{1}{2} \frac{i U k \hat{h} e^{-\kappa H}\left(\kappa-n_{2}+\frac{g^{\prime}}{U^{2}} \frac{\kappa^{2}}{k^{2}}\right)}{\kappa \cosh (\kappa H)+\left(n_{2}-\frac{g^{\prime}}{U^{2}} \frac{\kappa^{2}}{k^{2}}\right) \sinh ((\kappa H)},
$$

if $|k|>l_{2}$.

Using (8) and (25), the Fourier transform of the pressure perturbation may be written in terms of $c_{1}$ and $d_{1}$ as

$$
\hat{p}(z=0)=\frac{i \rho_{0} k}{\kappa} U\left(c_{1}-d_{1}\right)
$$

where (27) or (28) may be used, depending on the wavenumber range considered. The drag may be split in the same way as in (15), with $D_{I}$ defined in a similar way, but now with

$$
D_{L}=8 \pi^{2} \operatorname{Im} \int_{-\infty}^{+\infty} \int_{l_{2}}^{+\infty} k \hat{p}(z=0) \hat{h}^{*} d k d l
$$

since no upper limit on $k$ is imposed by the solution (25).

Using (27)-(29), the definition of $D_{I}$ in (15) and (30), $D_{I}$ and $D_{L}$ may be calculated explicitly, yielding

$$
D_{I}=8 \pi^{2} \rho_{0} U^{2} \int_{-\infty}^{+\infty} \int_{0}^{l_{2}} \frac{k^{3} m_{2}|\hat{h}|^{2}}{\left[\kappa \cosh (\kappa H)-\frac{g^{\prime} \kappa^{2}}{U^{2} k^{2}} \sinh (\kappa H)\right]^{2}+m_{2}^{2} \sinh ^{2}(\kappa H)} d k d l
$$

$$
D_{L}=-8 \pi^{2} \rho_{0} U^{2} \operatorname{Im} \int_{-\infty}^{+\infty} \int_{l_{2}}^{+\infty} \frac{k^{3}|\hat{h}|^{2}}{\kappa} \frac{\kappa \sinh (\kappa H)+\left(n_{2}-\frac{g^{\prime} \kappa^{2}}{U^{2} k^{2}}\right) \cosh (\kappa H)}{\kappa \cosh (\kappa H)+\left(n_{2}-\frac{g^{\prime} \kappa^{2}}{U^{2} k^{2}}\right) \sinh (\kappa H)} d k d l
$$


Equations (31) and (32) may be normalized by the drag that would be exerted on the orography if the upper layer extended down to the surface (as in Teixeira et al. 2013b), namely (by analogy with (19))

$$
D_{0}=\frac{\pi}{4} \rho_{0} U^{2} l_{2} a h_{0}^{2}
$$

and the integrand in (32) may be simplified using contour integration, also as in Teixeira et al. (2013b) but extended to 3D, and following even more closely than in the preceding subsection the treatment of Teixeira et al. (2017), to obtain

$$
\frac{D_{I}}{D_{0}}=64 \pi \frac{1}{l_{2}^{\prime}}\left(\frac{a}{H}\right)^{3} \int_{0}^{+\infty} \int_{0}^{l_{2}^{\prime}} \frac{k^{\prime 3} m_{2}^{\prime}\left|\hat{h}^{\prime}\right|^{2}}{\left(\kappa^{\prime} \cosh \kappa^{\prime}-\mathrm{Fr}^{-2} \frac{\kappa^{\prime 2}}{k^{\prime 2}} \sinh \kappa\right)^{2}+m_{2}^{\prime 2} \sinh ^{2} \kappa} d k^{\prime} d l^{\prime}
$$

where $\mathrm{Fr}=U /\left(g^{\prime} H\right)^{1 / 2}$ is the Froude number of the flow, and

$$
\frac{D_{L}}{D_{0}}=64 \pi^{2} \frac{1}{l_{2}^{\prime}}\left(\frac{a}{H}\right)^{3} \int_{0}^{\infty} \frac{k_{L}^{\prime 2}\left|\hat{h}^{\prime}\right|^{2}\left(k_{L}^{\prime}\right)\left\{\left[\operatorname{Fr}^{-2} \frac{\kappa_{L}^{\prime 2}}{k_{L}^{\prime 2}}-n_{2}^{\prime}\left(k_{L}^{\prime}\right)\right]^{2}-\kappa_{L}^{\prime 2}\right\}}{\left\{\kappa_{L}^{\prime 2}\left[1+\operatorname{Fr}^{-2} \frac{l^{\prime 2}}{k_{L}^{\prime 4}}+\frac{\kappa_{L}^{\prime 2}}{k_{L}^{\prime 2} n_{2}^{\prime}\left(k_{L}^{\prime}\right)}\right]+\left[1+\frac{l^{\prime 2}}{k_{L}^{\prime 2}}+n_{2}^{\prime}\left(k_{L}^{\prime}\right)-\operatorname{Fr}^{-2} \frac{\kappa_{L}^{\prime 2}}{k_{L}^{\prime 2}}\right]\left[\operatorname{Fr}^{-2} \frac{\kappa_{L}^{\prime 2}}{k_{L}^{\prime 2}}-n_{2}^{\prime}\left(k_{L}^{\prime}\right)\right]\right\}} d l^{\prime}
$$

where $k_{L}^{\prime}$ is the resonant wavenumber and $\kappa_{L}^{\prime}=\left(k_{L}^{\prime 2}+l^{\prime 2}\right)^{1 / 2}$. The resonance condition that this wavenumber must satisfy is obtained by imposing that the denominator of the integrand in (32) be zero, which gives

$$
\tanh \kappa_{L}^{\prime}=\frac{\kappa_{L}^{\prime}}{\operatorname{Fr}^{-2} \frac{\kappa_{L}^{\prime 2}}{k_{L}^{\prime 2}}-n_{2}^{\prime}\left(k_{L}^{\prime}\right)}
$$

Equations (34)-(35) may be compared with their 2D counterparts, derived by Teixeira et al. (2013b) (their Eqs. (20) and (25)). Differences account for the effects associated with the transition from a 2D to a 3D geometry, namely the fact that $D_{I}$ is given by a double integral and $D_{L}$ by a single integral. In (35), and unlike in (21), there is no sum, since there is at most one resonant wave mode for $k$ (keeping $l$ fixed). Despite that, as in (21) there are an infinite number of resonant modes as $l$ varies continuously.

As in the previous section, the vertical velocity perturbation may be calculated from (22), but now using also (25). This gives in the lower layer

$$
w_{1}(x, y, z)=2 \operatorname{Re} \int_{0}^{+\infty} \int_{0}^{+\infty}\left\{c_{1}(k, l)\left[e^{i(k x+l y-\kappa z)}+e^{i(k x-l y-\kappa z)}\right]+d_{1}(k, l)\left[e^{i(k x+l y+\kappa z)}+e^{i(k x-l y+\kappa z)}\right]\right\} d k d l
$$

If a procedure entirely similar to that used to derive (24) is followed, the vertical velocity perturbation associated only with the resonant trapped lee wave modes is given by

$\frac{w_{L}(x, y, z)}{U\left(\frac{h_{0}}{a}\right)}=-4 \pi\left(\frac{a}{H}\right)^{3} \int_{0}^{+\infty} \frac{k_{L}^{\prime} \hat{h}^{\prime}\left(k_{L}^{\prime}\right)\left\{\left[\operatorname{Fr}^{-2} \frac{\kappa_{L}^{\prime 2}}{k_{L}^{\prime \prime}}-n_{L}^{\prime}\left(k_{L}^{\prime}\right)\right]^{2}-\kappa_{L}^{\prime 2}\right\} \sinh \left(\kappa_{L}^{\prime} z^{\prime}\right)\left[\cos \left(k_{L}^{\prime} x^{\prime}+l^{\prime} y^{\prime}\right)+\cos \left(k_{L}^{\prime} x^{\prime}-l^{\prime} y^{\prime}\right)\right]}{\left\{\left[1+n_{2}^{\prime}\left(k_{L}^{\prime}\right)+\frac{l^{\prime 2}}{k_{L}^{\prime 2}}-\operatorname{Fr}^{-2} \frac{\kappa_{L}^{\prime 2}}{k_{L}^{\prime 2}}\right]\left[\operatorname{Fr}^{-2} \frac{\kappa_{L}^{\prime 2}}{k_{L}^{\prime 2}}-n_{2}^{\prime}\left(k_{L}^{\prime}\right)\right]+k_{L}^{\prime 2}\left[1+\frac{\kappa_{L}^{\prime 2}}{k_{L}^{\prime 2} n_{L}^{\prime}\left(k_{L}^{\prime}\right)}+\operatorname{Fr}^{-2} \frac{l^{\prime 2}}{k_{L}^{\prime 4}}\right]\right\}} d l^{\prime}$,

which again is only strictly applicable some distance downstream of the obstacle. Note that in (38), as in (35), it is not obvious that the denominator of the fraction cannot take the value zero, but that is the case, owing to the constraint imposed by (36). This means that the corresponding integrals over $l^{\prime}$ do not have any singularities. 


\section{Results}

The results obtained above from linear theory are explored in this section in parameter space by comparison with the corresponding 2D results presented by Teixeira et al. (2013a, 2013b), and verified against numerical simulations of similar flows.

\subsection{Atmosphere of Scorer (1949)}

Equation (20), giving the internal wave drag for the atmosphere of Scorer (1949), is normalized by (19) (using $l_{1}$ as the reference Scorer parameter) because this makes the drag oscillate around a value of 1 for hydrostatic flow as $l_{1} H$ increases. The normalized drag depends on $l_{1} H, l_{2} / l_{1}$ and $l_{1} a$, and will be presented next as a function of these variables, as in Teixeira et al. (2013a). The advantage of normalizing $H$ using $l_{1}$ is that it is possible to assign the location of maxima in the drag to semi-integer values of $l_{1} H / \pi$, as shown below. The appropriateness of normalizing $a$ using $l_{1}$ may seem questionable, as discussed in the next section, but is adopted here for consistency with Teixeira et al. (2013a). Equation (21) gives the trapped lee wave drag, normalized in a similar way, which is a function of the same dimensionless parameters.

Figure 2 shows the normalized internal wave drag $D_{I} / D_{0}$ given by (20) (Figure 2(a)), the normalized trapped lee wave drag $D_{L} / D_{0}$ given by (21) (Figure 2(b)), and the total drag, which is a sum of the two (Figure 2(c)), as a function of $l_{1} H$ and $l_{2} / l_{1}$ for $l_{1} a=10$. Figures 3 and 4 show the same, but for $l_{1} a=5$ and $l_{1} a=2$ instead, respectively. From Figure 2 to Figure 4, the flow becomes progressively more non-hydrostatic. These figures can be directly compared with Figures 3, 4 and 5 of Teixeira et al. (2013a), as even the contour lines used in the graphs are similar. A preliminary comment to make is that, unlike in Teixeira et al. (2013a), it does not make sense to show the number of trapped lee wave modes and their wavelengths in the present 3D case. Although discrete resonant modes, defined by (18), do exist for the wavenumber along the $x$ direction, $k_{j}$, for a given value of $l, l$ varies continuously, making $m_{1}$ also vary continuously and become potentially indefinitely large. This causes a potentially infinite number of resonant modes, where $k_{j}$ varies continuously with $l$, although for sufficiently large values of $l$ the amplitude of these modes should be negligible, given the weak orographic forcing that necessarily exists at those wavenumbers.

Beginning with the similarities between the 3D and 2D flows, Figures 2-4 show that maxima of both $D_{I} / D_{0}$ and $D_{L} / D_{0}$ occur roughly for $l_{1} H / \pi=0.5+n$, where $n$ is an integer. This results from the fact that for these heights of the interface between the two layers there is constructive interference in the lower layer between waves whose energy is propagating upward and those whose energy is propagating downward. Destructive interference occurs roughly for $l_{1} H / \pi=n$ instead. All these waves may either be partially reflected at $z=H$ (contributing in that case to $D_{I}$ ) or totally reflected at $z=H$ (i.e., trapped) (contributing then to $D_{L}$ instead). Concerning the dependence on $l_{2} / l_{1}, D_{I} / D_{0}$ attains a maximum at relatively low values of $l_{2} / l_{1} \approx 0.2-0.4$ (for which a substantial fraction of the non-trapped waves are partially reflected), whereas $D_{L} / D_{0}$ always attains its maximum at $l_{2} / l_{1}=0$ (corresponding to a situation where there is total reflection for all waves, and hence no internal waves exist). The total drag $D_{I} / D_{0}+D_{L} / D_{0}$ always attains its maximum for $l_{2} / l_{1}=0$, and tends to decrease with $l_{1} a$, as can be seen in Figures 2-4. However the fraction $D_{L} / D_{I}$ increases as $l_{1} a$ decreases, which makes sense physically because trapped lee waves tend to be favoured when the flow is substantially non-hydrostatic.

However, there are some differences between the present 3D case and the 2D case addressed by Teixeira et al. (2013a). Both $D_{I} / D_{0}$ and $D_{L} / D_{0}$ have a lower maximum magnitude in Figures 2-4 than in Teixeira et al. (2013a), and the magnitude of the drag peaks decreases markedly as $l_{1} H$ increases, something that does not occur in Teixeira et al. (2013a) to such a large extent. These two aspects are due to wave dispersion, which is weaker in 2D flows than in 3D flows, because so-called 'directional dispersion', associated with the fact that horizontal wavenumber vectors may point in all directions, only exists in 3D (note the similarity with, e.g., Teixeira et al. (2008)). Since, as the energy of the waves existing in the lower layer propagates upward and downward between reflections, their intensity decreases due to this effect, this explains not only the globally smaller magnitude of the drag, but also its decrease as $l_{1} H$ 

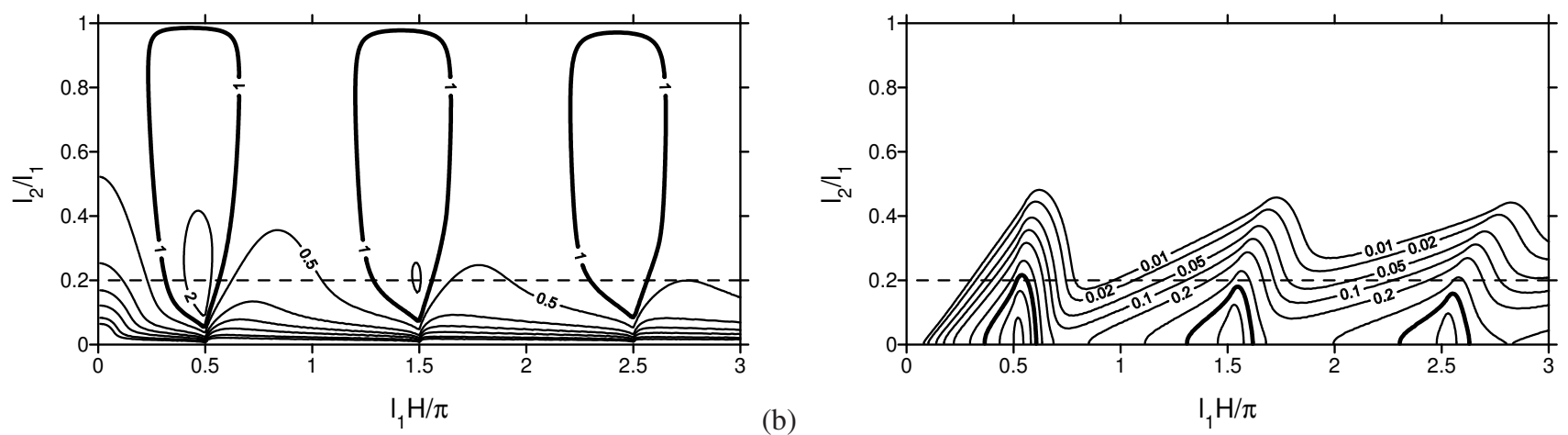

(a)

$\mathrm{I}_{1} \mathrm{H} / \pi$

(b)

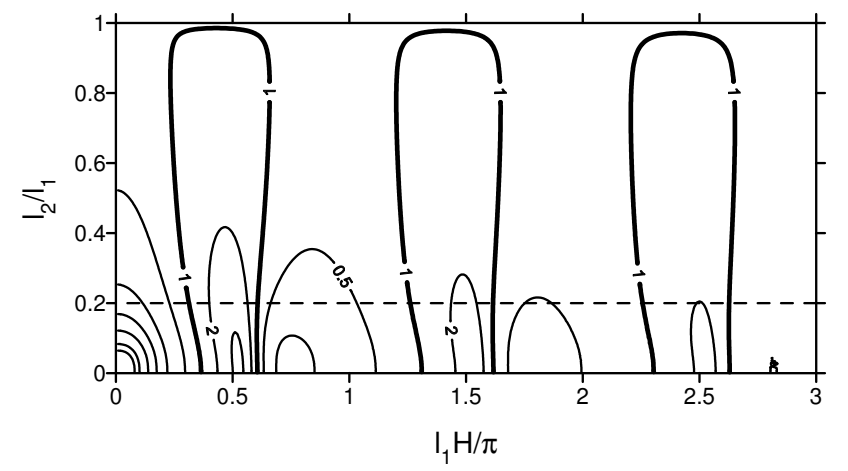

Figure 2. Normalized drag as a function of $l_{1} H$ and $l_{2} / l_{1}$ for $l_{1} a=10$. (a) Internal wave drag, given by (20); (b) trapped lee wave drag, given by (21); (c) total drag (sum of the two). Labelled solid contours have values $0.01,0.02,0.05,0.1,0.2 .0 .5$, and so on (thick solid contour corresponds to 1 ), and the dashed lines denote $l_{2} / l_{1}=0.2$.

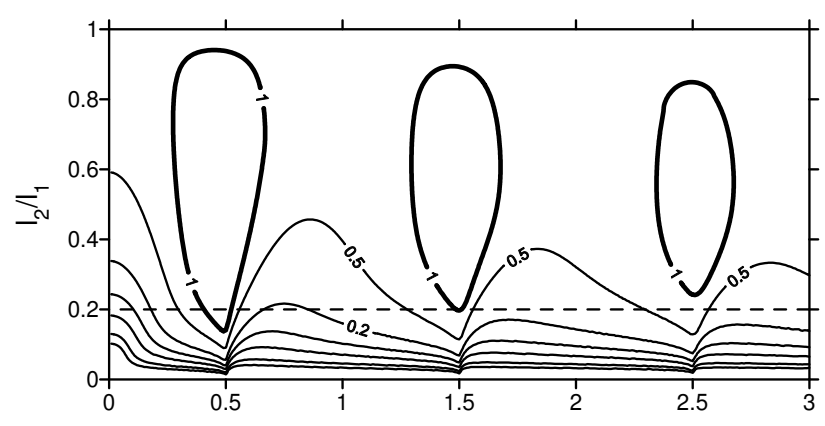

(a)

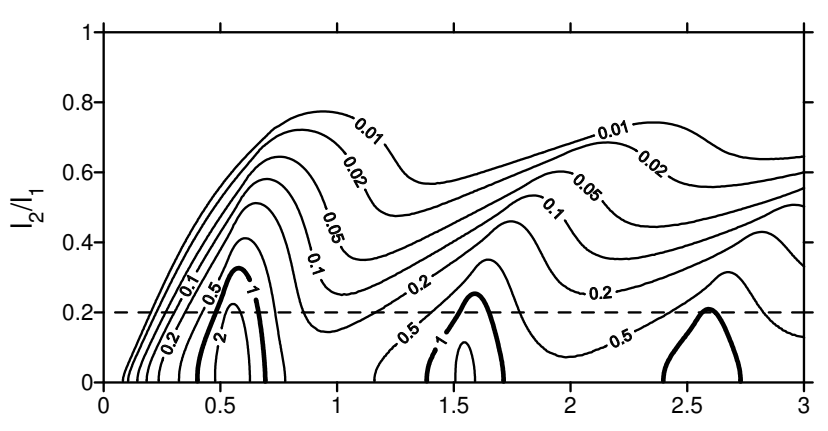

(b)
$\mathrm{I}_{1} \mathrm{H} / \pi$

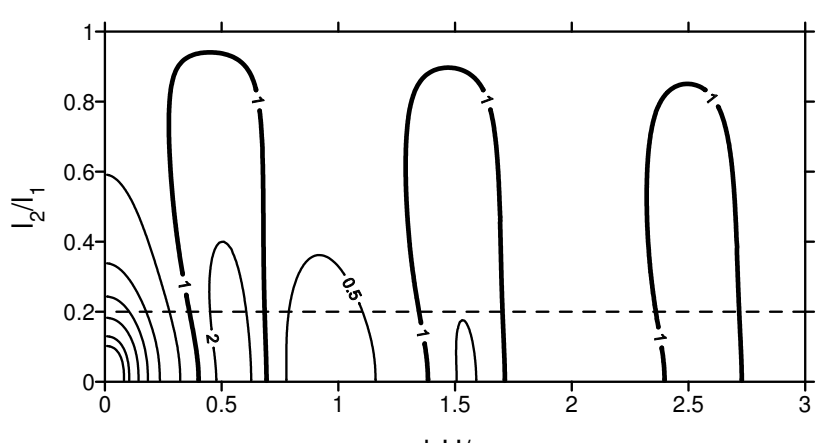

(c)

$$
\mathrm{I}_{1} \mathrm{H} / \pi
$$

Figure 3. Similar to Figure 2, but for $l_{1} a=5$.

increases. More importantly, since the trapped lee waves do not have a discrete spectrum in a 3D geometry but rather a continuous one, in Figures 2(b)-4(b) (unlike in Figures 3(b)-4(b) of Teixeira et al. (2013a)) the maxima of the trapped lee wave drag are not isolated. Rather, non-zero values of $D_{L} / D_{0}$ occur throughout the whole range of $l_{1} H / \pi$ for all values of $l_{1} a$ (although some of the gaps existing in Figures 3(b)-4(b) of Teixeira et al. (2013a) may be an artefact of the contour values adopted). More definite evidence is that, although no trapped lee wave drag is possible for any wave mode for $l_{1} H / \pi<0.5+n$ in Figures 3(b)-5(b) of Teixeira et al. (2013a), no such 


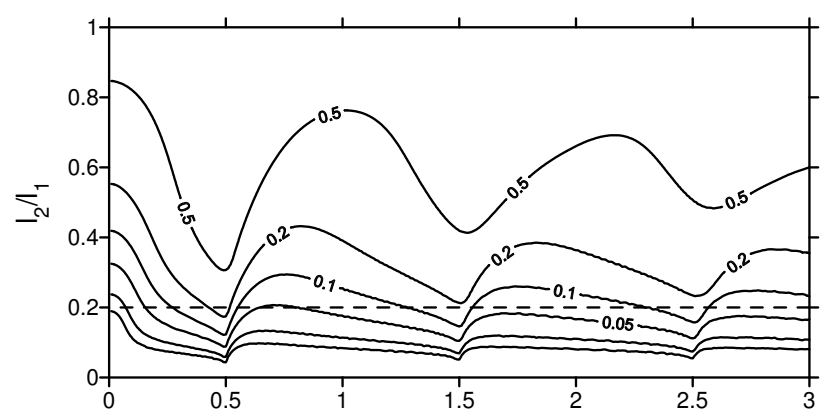

(a)

$\mathrm{I}_{1} \mathrm{H} / \pi$

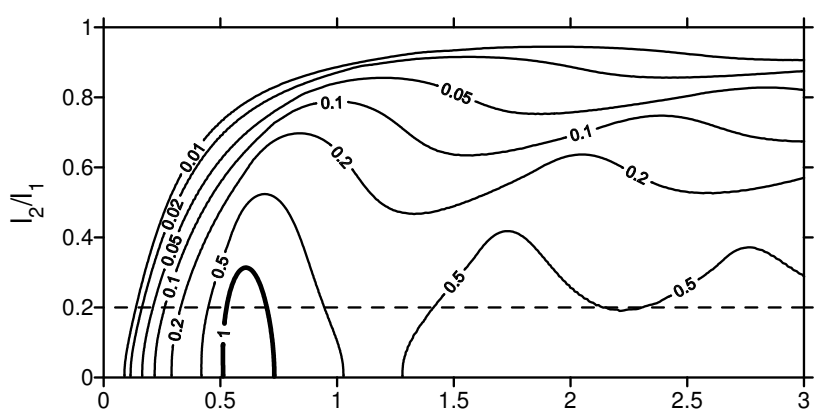

(b)

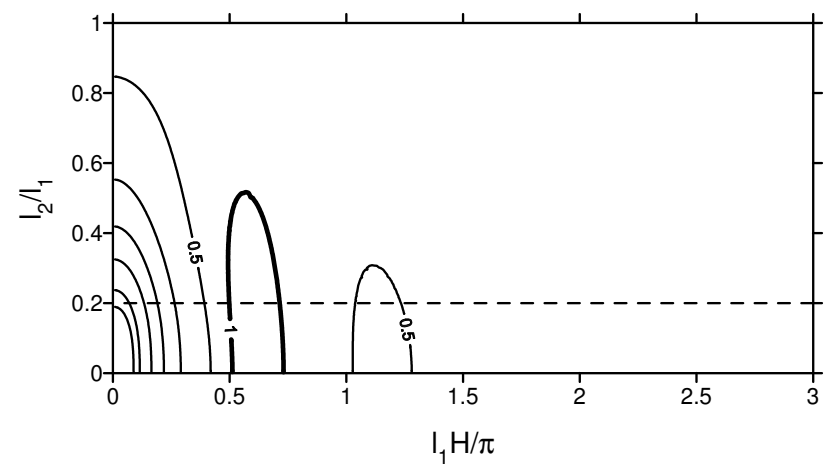

Figure 4. Similar to Figure 2, but for $l_{1} a=2$.

bound exists in Figures 2-4 of the present study (as is clearly shown by the non-zero values of $D_{L} / D_{0}$ for $l_{1} H / \pi<0.5$ ). This happens because resonant wave modes have a continuous distribution, as noted above.

Despite these differences, it remains true that, as in Teixeira et al. (2013a), both $D_{I} / D_{0}$ and $D_{L} / D_{0}$, and of course the total drag (their sum), reach values substantially higher than 1 . These values would be even larger if $D_{I}$ and $D_{L}$ had been normalized by the drag that would apply if the upper layer extended down to the surface $(H=0)$ (i.e., using $l_{2}$ instead of $l_{1}$ as the reference Scorer parameter), since this is smaller than $D_{0}$, as defined by (19), by a factor of $l_{2} / l_{1}$. What this means is that the trapped lee wave drag may not only be comparable or even higher than the internal wave drag, but it can also be larger than the hydrostatic reference value that is currently used in parametrizations. The trapped lee wave drag is applied on the atmosphere at low levels and downstream of the orography, an effect that is currently not taken into account in most parametrizations.

The parameter space covered by Figures 2-4 is too vast to be tested using numerical simulations. For that reason, the same approach as adopted in Teixeira et al. (2013a) will be used to test a sample of the results given by linear theory, namely assuming that $l_{2} / l_{1}=0.2$. This value is chosen because, while not being geophysically absurd (it may represent, for example, a situation with a strongly stable nocturnal boundary layer beneath a more neutral residual layer), it makes the drag retain a very substantial modulation with $l_{1} H$, being appreciably amplified with respect to its hydrostatic reference value. An example of such a stable layer, with Brunt-Väisälä frequency $N_{1} \approx 0.05 \mathrm{~s}^{-1}$ and thickness $H \approx 100 \mathrm{~m}$, is shown in Figure 4 of Cassano (2014), from measurements taken over Antarctica in Winter.

Figure 5 shows $D_{I} / D_{0}$ given by (20) (dashed lines), $D_{L} / D_{0}$ given by (21) (dotted lines) and the sum of the two (solid lines) for $l_{2} / l_{1}=0.2$ as a function of $l_{1} H / \pi$ and the same values of $l_{1} a$ considered in Figures 2-4, namely $l_{1} a=10,5,2$ (Figures 5(a)-(c)) This corresponds to the cross sections marked by the dashed lines in Figures 2-4. The total drag is compared with that diagnosed from simulations of the NH3D nonlinear and non-hydrostatic 3D mesoscale numerical model (circles) using a dimensionless mountain height $l_{1} h_{0}=0.02$ (for more details about the numerical simulations see the Appendix). For the three cases considered, the agreement of the total drag given by linear theory and by the numerical model is remarkably good. In this representation, the magnitude of the drag maxima is clearer than in Figures 2-4, and it can be seen that the first maximum of the normalized total drag has magnitudes 

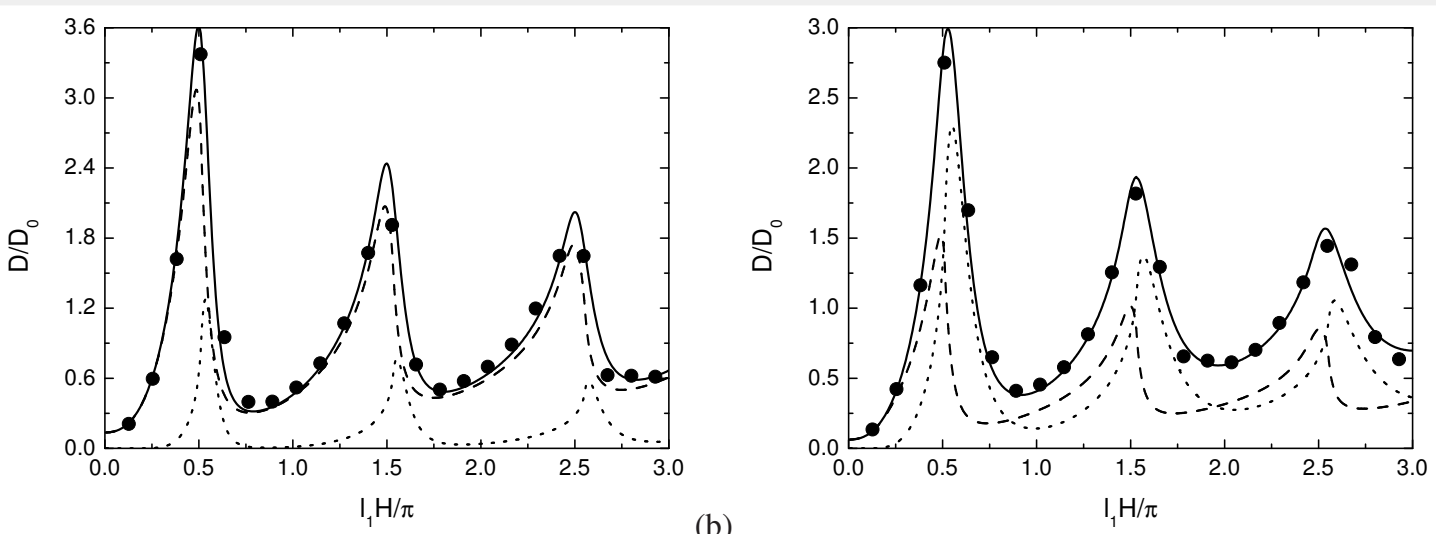

(a)

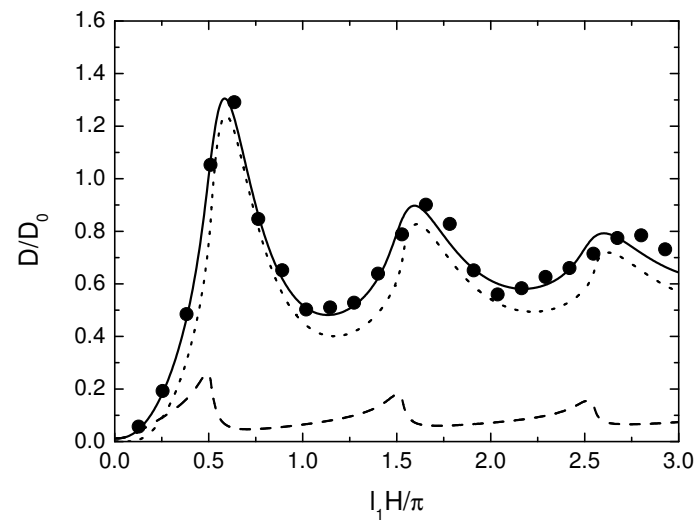

(c)

Figure 5. Normalized drag as a function of $l_{1} H$ for $l_{2} / l_{1}=0.2$. (a) $l_{1} a=10$; (b) $l_{1} a=5$; (c) $l_{1} a=2$. Dashed lines: internal wave drag, given by (20), dotted lines: trapped lee wave drag, given by (21), solid line: total drag (sum of the two); symbols: results from numerical simulations of the NH3D model for $l_{1} h_{0}=0.02$.

of $\approx 3.6, \approx 3.0$ and $\approx 1.3$ for $l_{1} a=10, l_{1} a=5$ and $l_{1} a=2$, respectively, against $\approx 5.5, \approx 5$ and $\approx 2.5$ in Figure 17 Teixeira et al. (2013a). The modulation of the drag as $l_{1} H / \pi$ increases also weakens in a more pronounced way, although this effect already exists in the 2D case (presumably because of non-directional wave dispersion).

A final aspect that can be noted when comparing Figure 5 with Figure 17 of Teixeira et al. (2013a) is that the trapped lee wave drag component is more dominant for the same values of $l_{1} a$. For example, in Figure 5(b) (for $l_{1} a=5$ ), the maximum magnitude of $D_{L} / D_{0}$ exceeds that of $D_{I} / D_{0}$, whereas in Figure 17 of Teixeira et al. (2013a) the opposite happens. One may speculate that this is a manifestation of the fact that non-hydrostatic effects are stronger in a 3D than in a $2 \mathrm{D}$ geometry, because the finite width of the orography is felt not only in one horizontal direction but in all directions. Alternatively, this can be viewed as another effect of directional dispersion.

A good way to check further whether the results from linear theory are reliable is by comparing the flow field itself with the results of numerical simulations. A component of the flow field that is potentially very sensitive is the trapped lee wave signature, which exists primarily downstream of the mountain, because it relies on fulfilment of the resonance condition (18). The non-trapped lee waves (i.e. internal waves) are expected to decay fairly fast downstream of the orography. Figure 6 shows a comparison of the normalized vertical velocity $w /\left(U h_{0} / a\right)$ downstream of the mountain for $l_{1} H=1.6$ (i.e. near the first drag maximum in Figure 5 ) at $z / H=0.875$, from numerical simulations (Figure 6(a),(c),(e)) and from linear theory, in the latter case only for the resonant trapped lee wave component (24) (Figure 6(b),(d),(f)), again for $l_{1} a=10,5,2$.

The first aspect to note is that the $w$ field downstream of the mountain superficially resembles a Kelvin ship wake (as noted before for a similar flow by, for example, Scorer and Wilkinson (1956) and for more complicated atmospheric profiles by Sawyer (1962) and Sharman and Wurtele (1983)). However, the dynamics and morphology of these waves is considerably more complicated than that of the surface waves addressed by Kelvin, since these are internal waves (propagating in the lower layer), may have multiple modes for a given lateral wavenumber $l$, and their dispersion relation depends not only on a horizontal wavenumber, but also on 

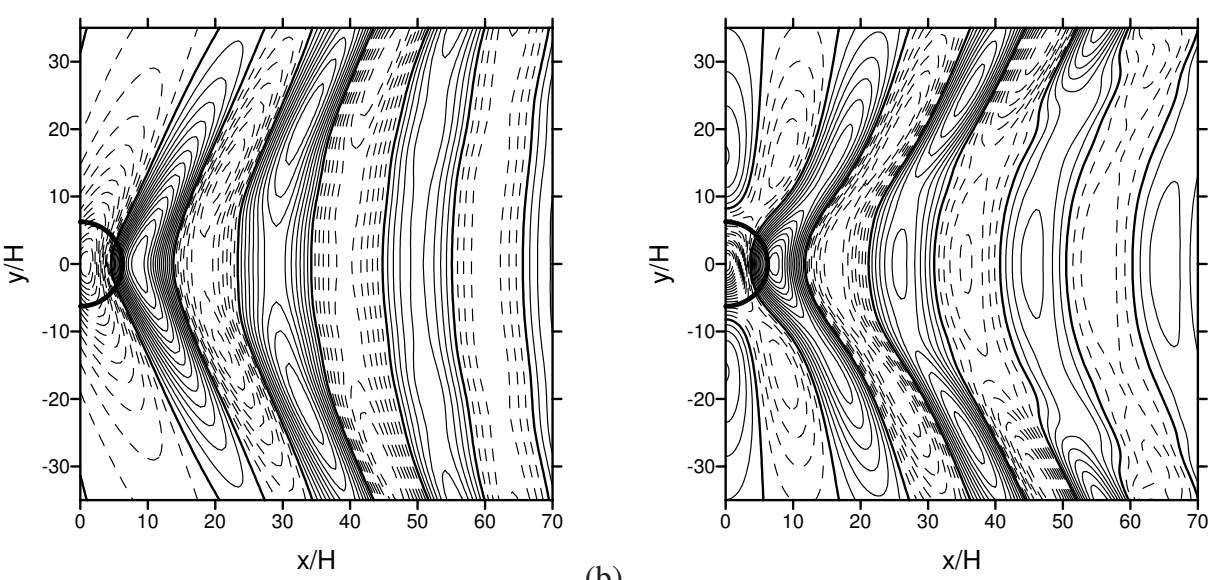

(a)

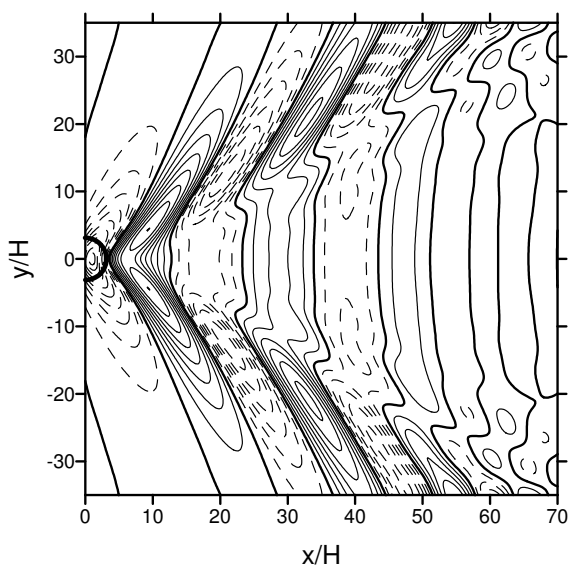

(c)

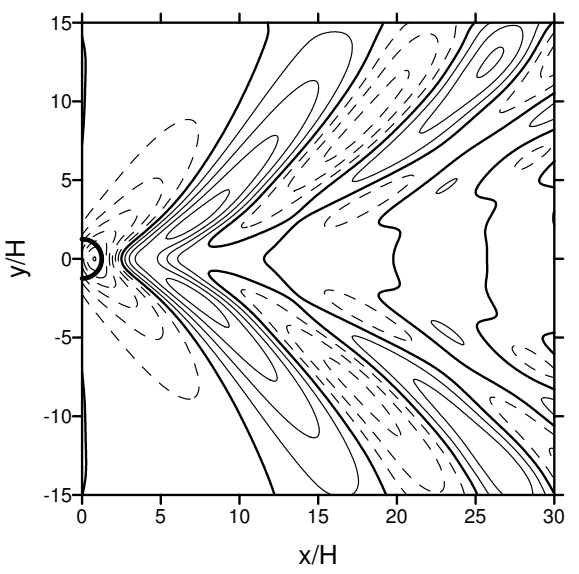

(b)

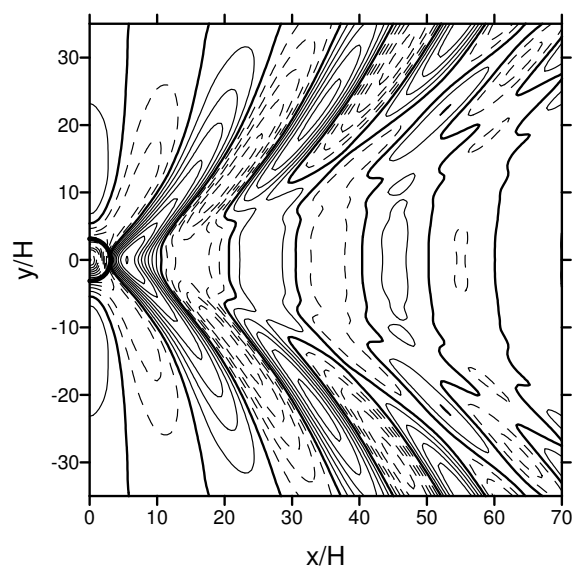

(d)

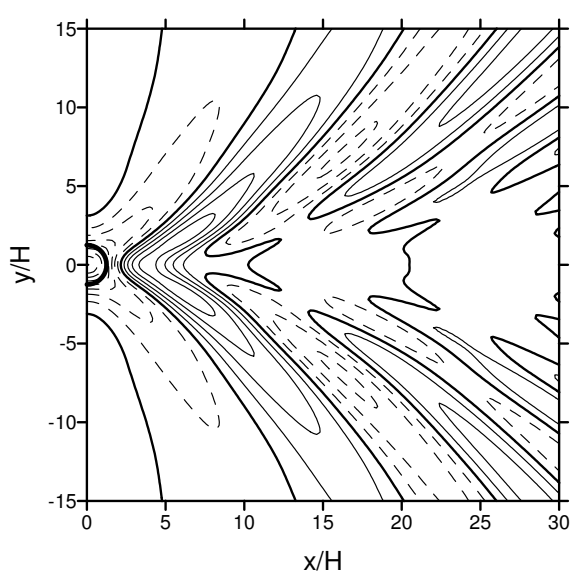

(e)

(f) contour spacing 0.2 ; (c) and (d) $l_{1} a=5$, contour spacing 0.2 ; (e) and (f) $l_{1} a=2$, contour spacing 0.1 . (a), (c) and (e) Numerical simulations of NH3D for $l_{1} h_{0}=0.02$; (b), (d) and (f) results from linear theory, given by (24). Solid contours: positive values, dashed contours: negative values. Thick circle: outline of the mountain half-width, $\left(x^{2}+y^{2}\right)^{1 / 2}=a$.

the vertical wavenumber $m_{1}$. This makes a quantitative interpretation of the wake angles more difficult, as discussed at length by Sharman and Wurtele (1983). Nevertheless, it is clear that the wavy wake is also roughly triangular, emanating from the mountain. A qualitative feature that can be deduced from the relationship between the phase speed and group speed of the waves (see schematic in Figure 68 of Lighthill (1978)) is that, the more non-hydrostatic the flow becomes the narrower the wake angle should be, because, as happens in interfacial waves, the group velocity differs more from the phase velocity (i.e. the waves become more dispersive). This behaviour can indeed be confirmed in Figure 6.

Because the trapped lee waves are internal and not interfacial waves, the fields are presented in Figure 6 for $z / H=0.875$, not $z=H$, as it can be shown theoretically that the amplitude of the trapped lee waves attains a maximum between $z / H=1 / 3$ and $z / H=1$. The 
fields are also presented as a function of $x / H$ and $y / H$, since all quantities included in the resonance condition (18) are normalized by $H$, which means that the resonant wavenumbers are independent of $a$.

The agreement between $w /\left(U h_{0} / a\right)$ in Figure 6(a),(c),(e) and Figure 6(b),(d),(f) is remarkably good, especially having in mind that the analytical solutions only take into account the part of the vertical velocity associated with trapped lee waves. The agreement of the numerical and analytical solutions is not as good nearer to the mountain, as would be expected, but it is still remarkable overall (this can be checked by counting the number of contours in each graph). The maxima attained by all fields are always slightly smaller (by a factor of $\approx 1.1-1.2$ ) in the numerical solutions, and the smallest-scale features are slightly smoothed (this is especially visible in the comparison between Figure 6(c),(e) and Figure 6(d),(f)), which may be speculatively attributed to computational diffusion. This attenuation might be counteracted, to a certain extent, by the amplification of $w$ due to the decay of density with height (which is not taken into account in linear theory), but this effect is relatively small at the height where the fields are plotted $(z=800 \mathrm{~m}$ in the numerical simulations).

\subsection{Atmosphere of Vosper (2004)}

Equation (34) gives the internal wave drag for the atmosphere of Vosper (2004), now normalized by the drag valid if the upper stratified layer extended down to the surface, (33), which is taken as the reference drag value, although other choices would be possible (Teixeira et al. 2017). Equation (35), on the other hand, gives the normalized trapped lee wave drag, in the present case, and as in Teixeira et al. (2013b), corresponding to waves that propagate at the temperature inversion, which acts essentially like a density interface. Both $D_{I} / D_{0}$ and $D_{L} / D_{0}$ depend on $\mathrm{Fr}, l_{2} H$ and $l_{2} a$, and are plotted as functions of these variables in the figures to be presented next. The choice of normalizing both $H$ and $a$ using $l_{2}$ is perhaps the most natural one, but $a / H$ could be used as an alternative measure of non-hydrostatic effects, as done in Teixeira et al. (2017), however the parameters presented above are kept for consistency with Teixeira et al. (2013b).

Figures 7-10 show $D_{I} / D_{0}, D_{L} / D_{0}$ and the total drag (the sum of the two) (panels (a), (b) and (c), respectively) as a function of Fr and $l_{2} H$ for $l_{2} a=5,2,1,0.5$. These values of $l_{2} a$ were chosen for consistency with Teixeira et al. (2013b), and correspond to cases where trapped lee wave drag has a considerable magnitude. However, the value $l_{2} a=0.2$, considered by Teixeira et al. (2013b) is ignored, as the corresponding drag would be too low in the present case. Figures 7-10 may be directly compared with the corresponding 2D results presented in Figures 3-6 of Teixeira et al. (2013b). As for the atmosphere of Scorer (1949), the flow becomes more nonhydrostatic as $l_{2} a$ increases, but the role of this parameter is different from that of $l_{1} a$, as will be discussed. In the present 3D flow geometry, there is either a single or no trapped lee wave mode in the $x$ direction, for a fixed $l$, with a resonant wavenumber prescribed by (36) (as was the case in Teixeira et al. (2013b)), but this depends the value of $l$. This resonance works in much the same way as for interfacial waves (Teixeira et al. (2017)), with the difference that the resonance condition is modified by stable stratification in the upper layer (cf. (36) and Eq. (19) of Teixeira et al. (2017); see also Sachsperger et al. (2015)). For a continuous distribution of $l$, the resonant wavenumber along $x, k_{L}$, thus also varies continuously, as for the atmosphere of Scorer (1949). This implies, in particular, that there is no upper bound on the value of Fr (which was 1 in the 2D case of Teixeira et al. (2013b)) for which trapped waves are allowed to exist.

Some similarities between the drag behaviour for 3D flow, illustrated in Figures 7-10, and for 2D flow, in Figures 3-6 of Teixeira et al. (2013b), may be noted. Firstly, both $D_{I} / D_{0}$ and $D_{L} / D_{0}$ show high values for low values of $l_{2} H$. This is because a strong enough influence of the orography on the upper layer, or on the interface, across the neutrally stratified lower layer, is necessary to produce substantial internal wave drag or trapped lee wave drag, respectively. Secondly, regions of high trapped lee wave drag are much more localized in parameter space than those of high internal wave drag, especially concerning the dependence on Fr and for the highest values of $l_{2} a$. Maxima of $D_{I} / D_{0}, D_{L} / D_{0}$ and of the total drag always occur near $\operatorname{Fr} \approx 1$ and $l_{2} H \approx 0$, but $D_{I} / D_{0}$ remains relatively 


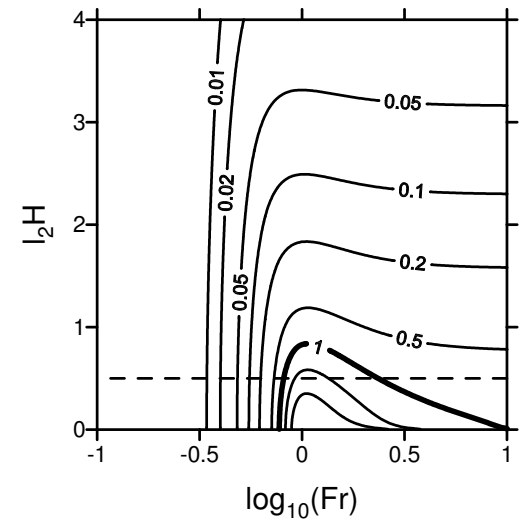

(a) $\log _{10}(\mathrm{Fr})$

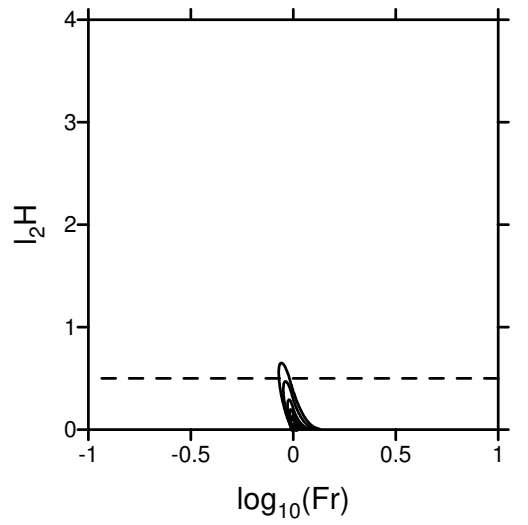

(b)

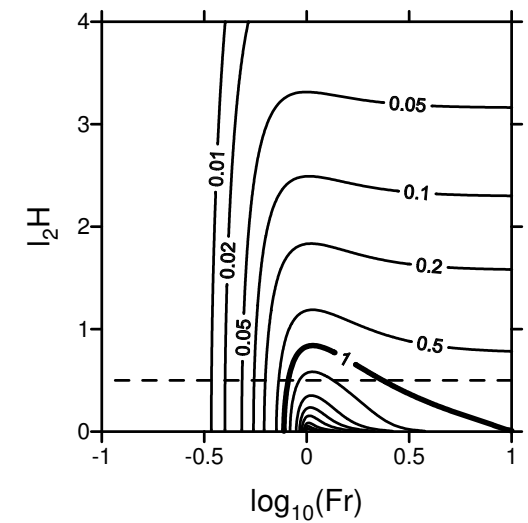

(c)

Figure 7. Normalized drag as a function of Fr and $l_{2} H$ for $l_{2} a=5$. (a) Internal wave drag, given by (34) ; (b) trapped lee wave drag, given by (35); (c) total drag (sum of the two). Labelled solid contours: $0.01,0.02,0.05,0.1,0.2,0.5$, and so on (thick solid contour corresponds to 1 ), and the dashed line denotes $l_{2} H=0.5$.

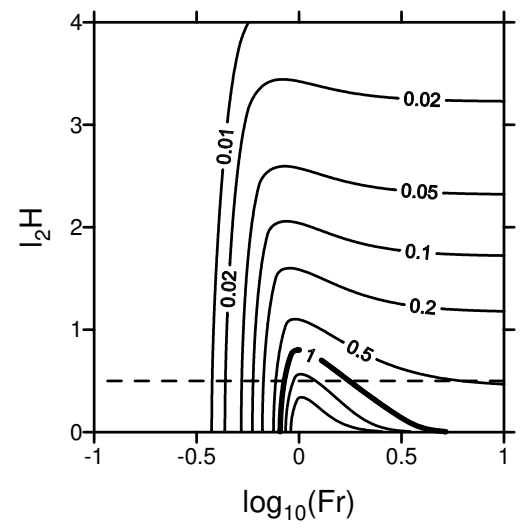

(a)

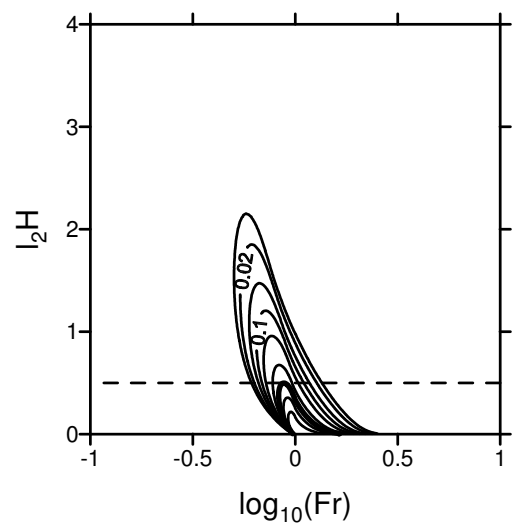

(b)

(c)

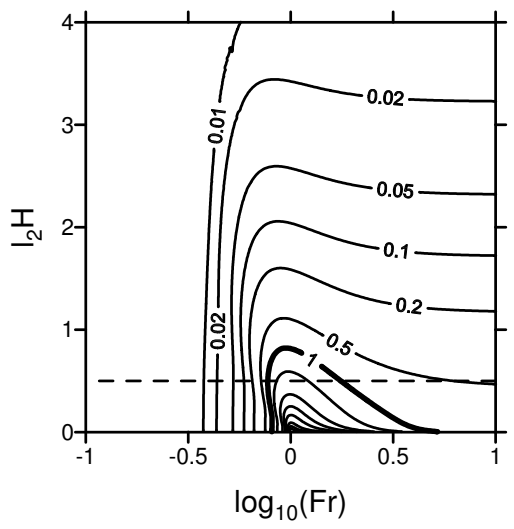

Figure 8. Similar to Figure 7, but for $l_{2} a=2$.

high for $\mathrm{Fr}>1$, whereas $D_{L} / D_{0}$ is concentrated around $\mathrm{Fr} \approx 1$, as this is associated with resonant conditions, where the phase speed of the trapped (interfacial) waves propagating upstream matches the downstream advection speed of the incoming flow. However, as explained in Figures 1 and 3 of Teixeira et al. (2017) for the slightly simpler case where the upper layer is not stratified, this matching can occur in $3 \mathrm{D}$ flow for $\mathrm{Fr}>1$, as opposed to $2 \mathrm{D}$ flow, where a requirement for it to happen is $\mathrm{Fr} \leq 1$. This is possible due to socalled 'divergent waves' (by analogy with Kelvin ship waves), which travel obliquely to the flow and that obviously do not exist in 2D. In Figures 7-10 it is clear that the total drag decreases with $l_{2} a$, but again the fraction $D_{L} / D_{I}$ increases as $l_{2} a$ decreases, i.e. the trapped lee waves become more dominant for more non-hydrostatic flow. As for the atmosphere of Scorer (1949), in the present $3 \mathrm{D}$ case the drag magnitude is smaller than in the 2D case of Teixeira et al. (2013b), because of directional wave dispersion, but both $D_{I} / D_{0}$ and $D_{L} / D_{0}$ (and consequently their sum) reach values substantially higher than 1 , stressing their potential importance in drag parametrizations.

To test the results from linear theory presented above, and following Teixeira et al. (2013b), a cross section of the graphs presented in Figures 7-10 for $l_{2} H=0.5$ is shown in Figure 11. This value of $l_{2} H$, on the one hand is low enough for the drag associated with it to be relevant, and on the other hand corresponds to geophysically reasonable conditions: if $l_{2}=10^{-3} \mathrm{~m}^{-1}$ (as assumed in the numerical simulations), this corresponds to $H=500 \mathrm{~m}$ which is a plausible height for the oceanic well-mixed boundary layer of a flow incident, for example, on a mountainous island.

Figure 11 shows $D_{I} / D_{0}, D_{L} / D_{0}$ and the total drag as a function of Fr, using the same line and symbol notation as in Figure 5, for $l_{2} H=0.5$ and $l_{2} a=5,2,1,0.5$ (panels (a)-(d)), being directly comparable with Figure 9 of Teixeira et al. (2013b), computed for 2D flow (minus panel (e) of that figure, for $l_{2} a=0.2$ ). The comparison of the total drag with results from numerical simulations of 


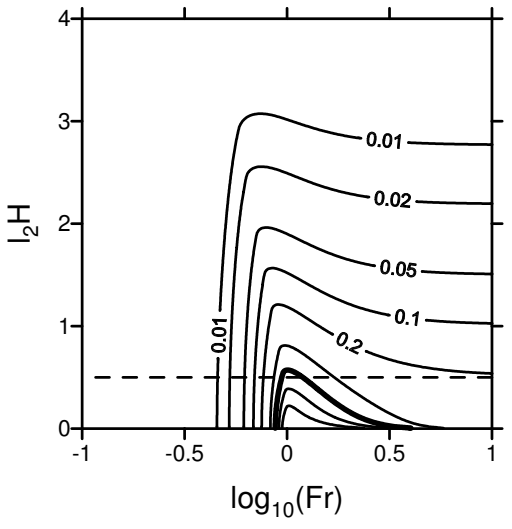

(a)

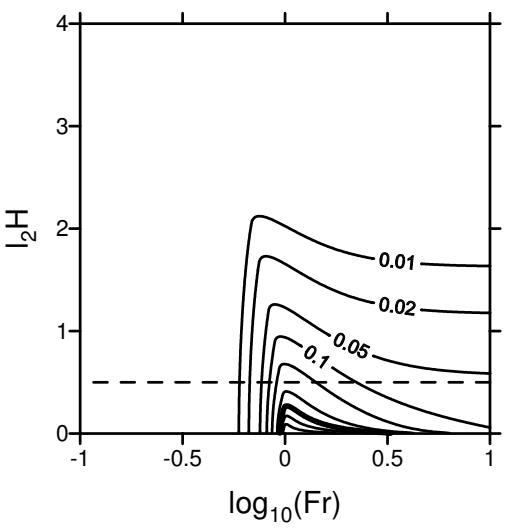

(a)

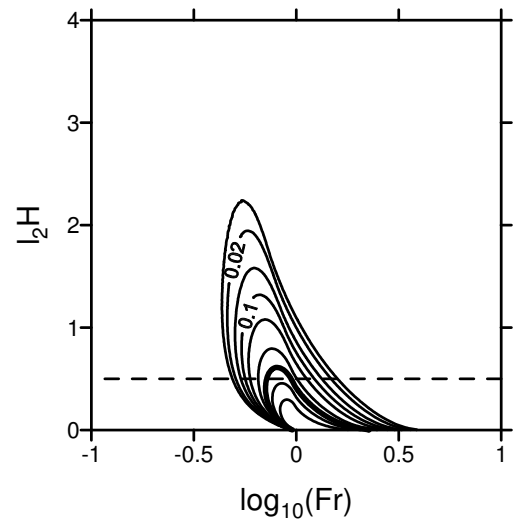

(b)

Figure 9. Similar to Figure 7, but for $l_{2} a=1$.

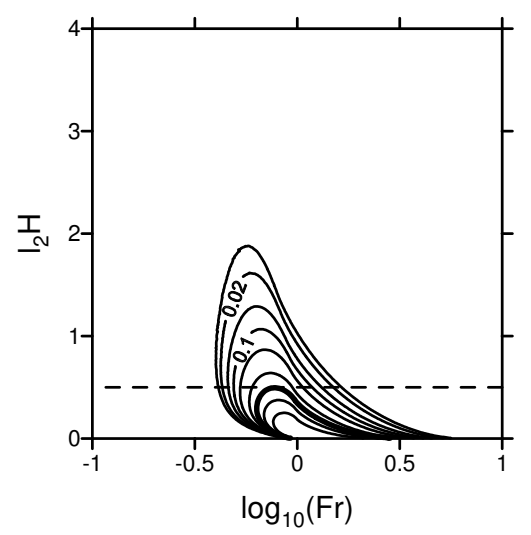

(b)

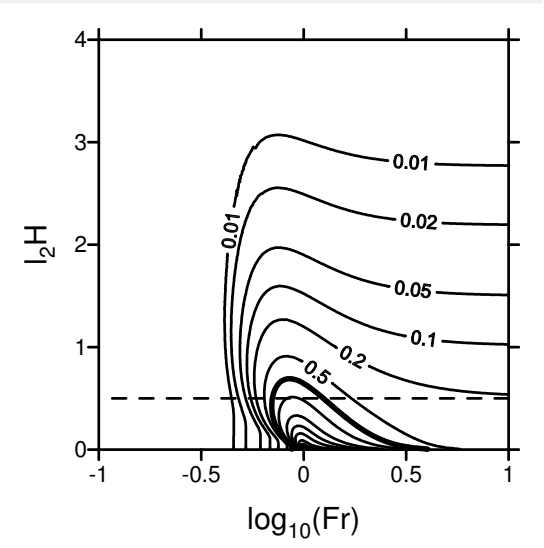

(c)

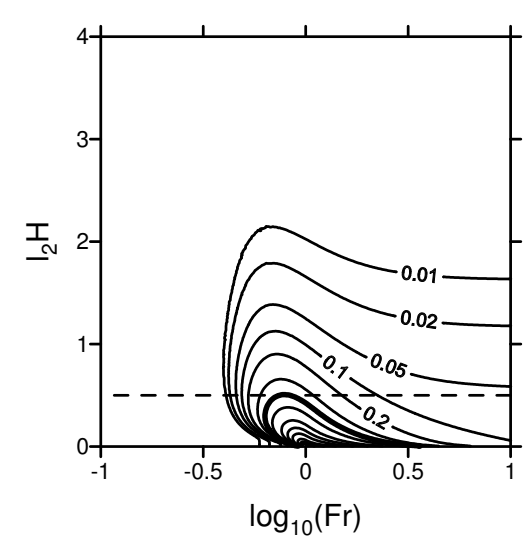

(c)

Figure 10. Similar to Figure 7, but for $l_{2} a=0.5$

the NH3D model (with $l_{2} h_{0}=0.01$ ) shows again very good agreement, except in relative terms to the left of the drag maximum, where rather low values are predicted, in which case linear theory somewhat underestimates the numerical simulation data. As for the atmosphere of Scorer (1949), the magnitude of the drag is substantially lower than in the 2D geometry used by Teixeira et al. (2013b). Namely, while in Teixeira et al. (2013b) the total drag maxima were $\approx 4, \approx 5, \approx 4$ and $\approx 3.5$ for $l_{2} a=5,2,1,0.5$, respectively, in Figure 11 the corresponding values are $\approx 2.75, \approx 3, \approx 2.25$ and $\approx 1$. This should be caused by the effects of directional wave dispersion. Although it is quite clear that non-zero values of the trapped lee wave drag extend to Fr $>1$ (see Figure 11(b)-(d)), a more pronounced dominance of $D_{L}$ over $D_{I}$ than in Figure 9 of Teixeira et al. (2013b) due to directional wave dispersion is not evident, as seen, for example, when Figure 11(c) is compared with Figure 9(c) of Teixeira et al. (2013b). The reasons for this behaviour are not apparent.

An aspect worth stressing is that, while in both flows treated in this and in the previous subsection the internal wave drag always tends to be maximized in the hydrostatic flow limit (where the trapped lee waves vanish), the trapped lee wave drag tends to be maximized for $l_{2} a$ of order 1 . Note that the values of $l_{2} a$ that correspond to the values of $l_{1} a$ used in Figure 5 (for $\left.l_{2} / l_{1}=0.2\right)$ are $l_{2} a=2,1,0.4$. These almost coincide with the values of $l_{2} a$ used in Figure 11 (except for the case with $l_{2} a=5$, where trapped lee waves are very weak). Hence, as long as the waves are able to propagate vertically in the lower layer, they become stronger as trapped lee waves when a larger faction of them is evanescent in the upper layer, which happens when the value of $l_{2} a$ is sufficiently low (an aspect noted by Teixeira et al. (2013b) and Yu and Teixeira (2015)). This is of course expected, since all waves would become evanescent in the upper layer (and thus trapped) in the limit $l_{2} \rightarrow 0$ (but this limit is not realistic in the atmosphere).

As for the atmosphere of Scorer (1949), the vertical velocity is a good indicator of whether the trapped lee waves are captured adequately by linear theory. Figure 12 shows fields of $w /\left(U h_{0} / a\right)$ from NH3D numerical simulations at $z / H=1$ (Figure 12 (a),(c),(e)) 

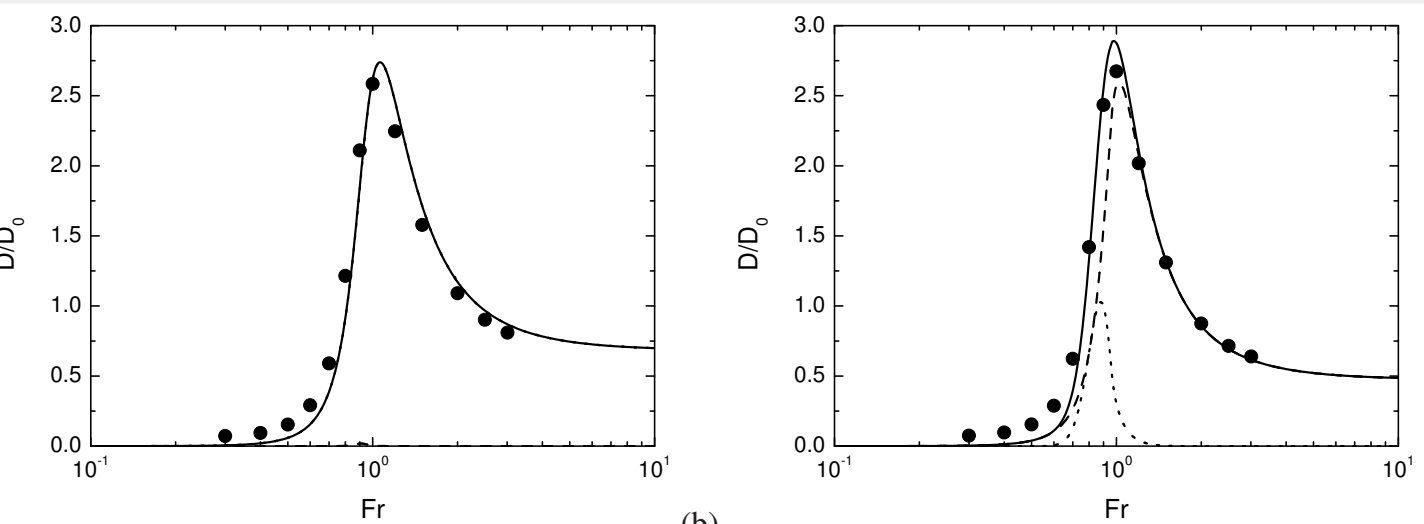

(a)

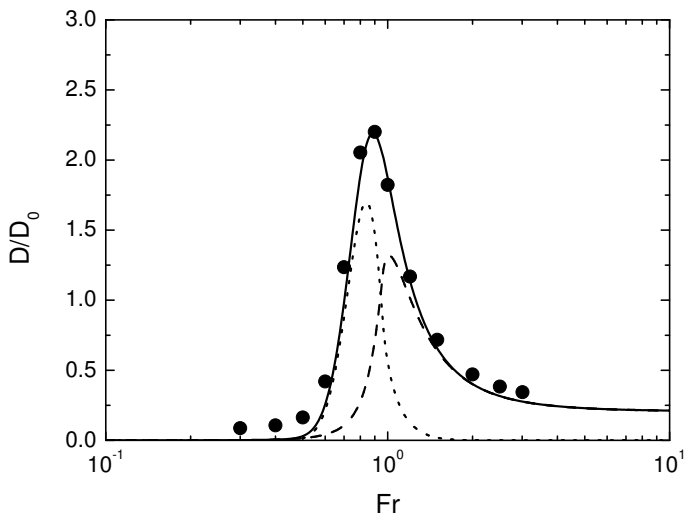

(b)

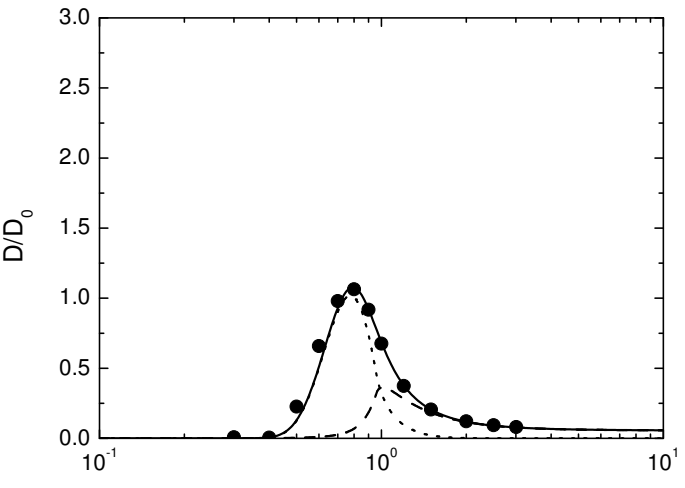

(d)

(c)

Figure 11. Normalized drag as a function of Fr for $l_{2} H=0.5$. (a) $l_{2} a=5$; (b) $l_{2} a=2$; (c) $l_{2} a=1$; (d) $l_{2} a=0.5$. Dashed line: internal wave drag, given by (34); dotted line: trapped lee wave drag, given by (35); solid line: total drag (sum of the two); symbols: results from numerical simulations of $\mathrm{NH}_{3 \mathrm{D}}$ for $l_{2} h_{0}=0.01$.

compared to the expression from linear theory for the vertical velocity associated only with trapped lee waves (38) (Figure 12(b),(d),(f)), as a function of $x / H$ and $y / H$ for $\mathrm{Fr}=0.8,0.9, l_{2} H=0.5$ and $l_{2} a=2,1,0.5$. The height $z / H=1$ was chosen because the waves under consideration are interfacial (as noted by Teixeira et al. (2013b)) and hence their amplitude peaks at the inversion between the two atmospheric layers. No fields were obtained for $l_{2} a=5$, as this corresponds to very weak trapped lee waves.

Also here, the wave pattern forms a triangular wake downstream of the mountain, reminiscent of a Kelvin ship wake. The resemblance is even closer, since these are interfacial waves, the only difference relative to the Kelvin ship wave problem being that the waves are modified by stratification in the upper layer (Sachsperger et al. 2015). The $w /\left(U h_{0} / a\right)$ field appears to have a 'purer' pattern than in Figure 6, with less small scale features (which is especially clear in panels (c)-(d) and (e)-(f) of both figures). This probably results from the fact that now there is only at most a single resonant wave mode for each $l$ value instead of multiple modes (cf. Sharman and Wurtele (1983)). Agreement between the numerical simulations and linear theory is remarkably good, except very near to the mountain, particularly in Figure 12(e)-(f), perhaps because these trapped lee waves are especially well resolved. The maxima and minima of the numerical simulations are still a factor of $\approx 1.2-1.3$ smaller than those from linear theory, and some sharper features are slightly smoothed in the simulations (see for example Figure 12(c)-(d)), likely due to numerical diffusion. The triangular shape of the wake has an angle that becomes narrower as the flow becomes more non-hydrostatic (i.e. $l_{2} a$ decreases), as can be seen going from panels (a)-(b) to (c)-(d) and (e)-(f) of Figure 12. This is again due to the fact that nearly hydrostatic waves are less dispersive (a result that is equally valid for internal and interfacial waves, cf. Teixeira et al. (2017)).

\section{Concluding remarks}

Mountain wave drag was evaluated explicitly using linear theory for two-layer atmospheres similar to those considered in the studies of Scorer (1949) and Vosper (2004). These may be viewed as idealized approximations to atmospheres affected by the stratification effects associated with either a stable boundary layer, or a well-mixed boundary layer, respectively. However, boundary layer effects on the 

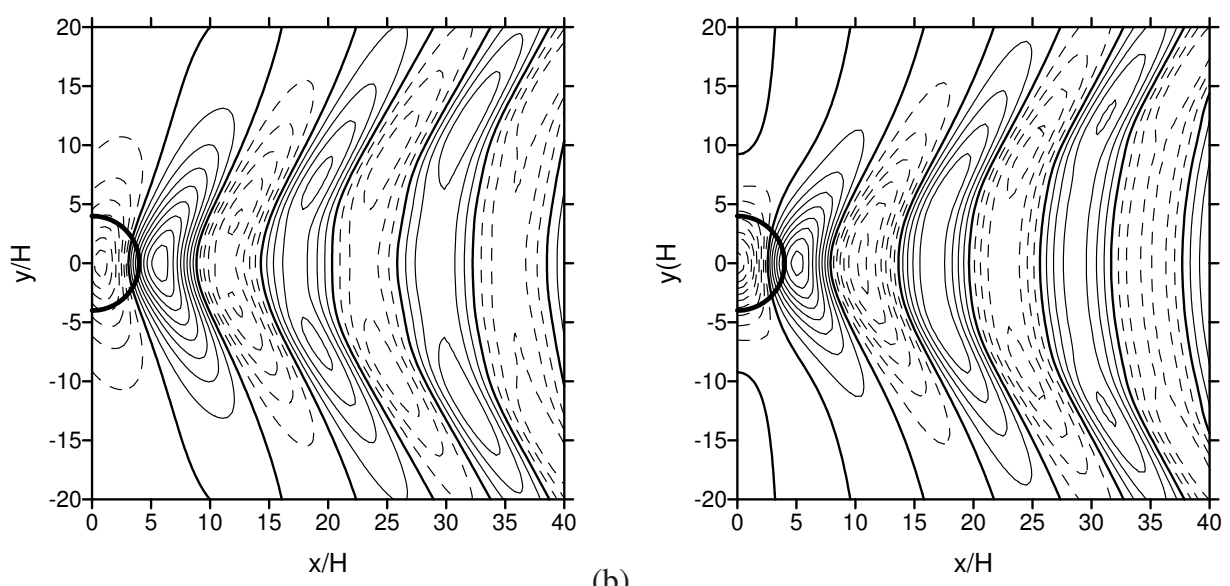

(a)

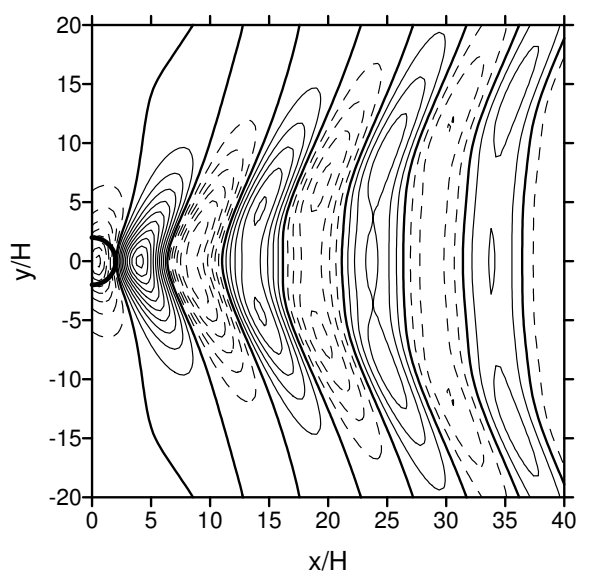

(c)

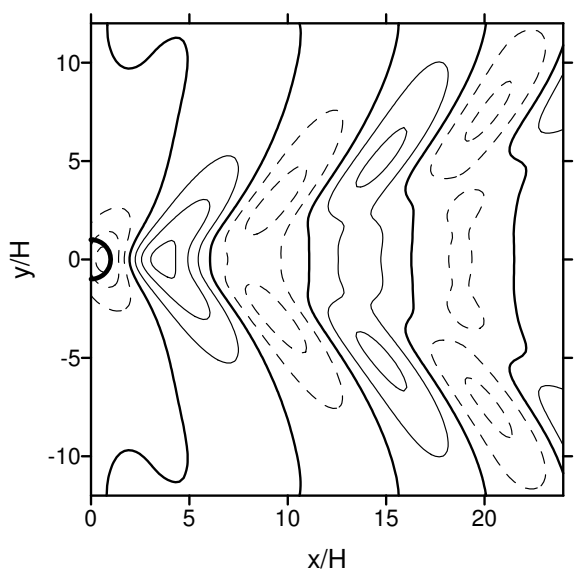

(b)

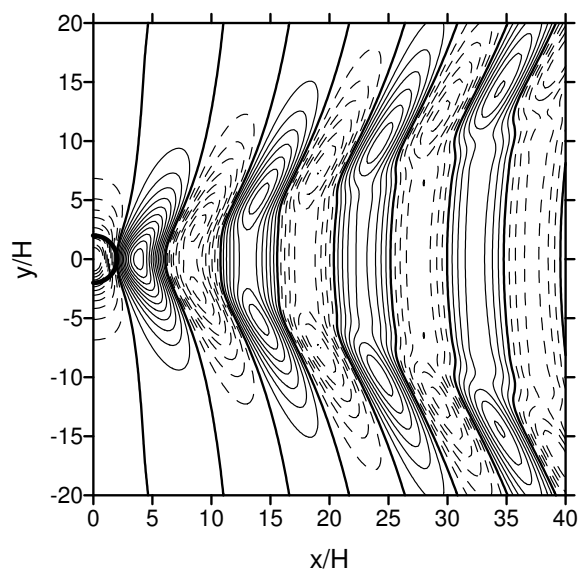

(d)

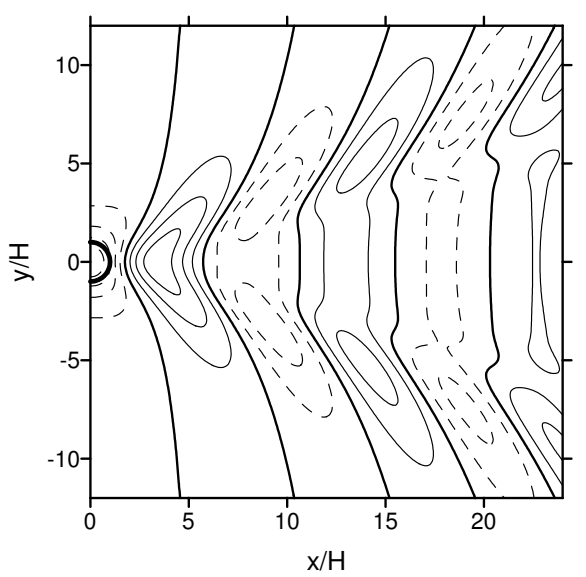

(e)

Figure 12. Normalized vertical velocity perturbation $w /\left(U h_{0} / a\right)$ for $l_{2} H=0.5$ at $z / H=1$. (a) and (b) $l_{2} a=2$ and Fr $=0.9$; (c) and (d) $l_{2} a=1$ and Fr $=0.8$; (e) and (f) $l_{2} a=0.5$ and $\mathrm{Fr}=0.8$. (a), (c) and (e) Numerical simulations from NH3D; (b), (d) and (f) results from linear theory, given by (38).

wind profile have been neglected, for simplicity. Both the drag associated with mountain waves that propagate vertically in the upper stratified layer and with trapped lee waves propagating either in the lower layer, or at the interface separating the two layers, have been calculated. But the main focus was on the trapped lee wave drag, for which a representation is currently missing in most orographic gravity wave drag parametrization schemes used operationally (e.g. Lott and Miller (1997)). For generic atmospheric profiles, where the wind and stratification vary continuously in the vertical, the dynamics of the trapped lee waves is exceedingly complex. However, for the idealized two-layer atmospheres considered here, it has been possible not only to calculate novel closed-form expressions for the trapped lee wave drag (expressed in terms of 1D integrals), but it is also much easier to understand the physical processes that explain the observed drag behaviour.

The behaviour of the trapped lee wave drag (and also of the drag associated with vertically propagating waves) was explored systematically in parameter space, and in more detail for selected values of the input parameters that potentially maximize its practical 
impact in parametrizations. This showed that, although the drag is lower for an axisymmetric obstacle than for 2D mountains, even as a fraction of its reference hydrostatic value, in this 3D flow configuration the trapped lee wave drag can still be a substantial fraction of the drag associated with waves that propagate vertically, sometimes even exceeding it. More importantly, both of these drag components may exceed the reference hydrostatic value for a uniformly stratified atmosphere by a factor considerably higher than 1 in the resonant conditions conducive to trapped lee waves, when the flow is tuned for constructive wave interference.

While the drag associated with vertically propagating waves is exerted on the atmosphere at high levels (often above the tropopause), the trapped lee wave drag, as represented in the present study, must be exerted on the atmosphere either in the lower layer (i.e. inside the thermal boundary layer) or at the interface between the two layers (i.e. at the top of the boundary layer). This makes it overlap spatially with turbulent form drag, for which it may be mistaken in the parametrization calibration process that is necessary to optimize the performance of weather prediction models (via, for example, a so-called 'long tail formulation' of turbulent form drag) (Steeneveld et al. 2008). Given the shortcomings of these calibration procedures, which improve certain metrics quantifying the skill of those models while degrading others, there seems to be much scope for developing representations of trapped lee wave drag separate from those of turbulent form drag (as seems physically reasonable), which may help to alleviate existing forecast biases and physical inconsistencies in models.

The present study aims to give a first contribution towards that aim. Before this is feasible, however, some improvements of the model calculations developed here may be necessary. For example, to represent the horizontal anisotropy that forms an essential part of a realistic orography representation, it would be useful to extend these calculations to mountains with an elliptical horizontal crosssection, as assumed in drag parametrizations. This may be straightforwardly done by using a coordinate transformation similar to those adopted by Phillips (1984), or Teixeira and Miranda (2009) and Teixeira and Yu (2014). These previous studies suggest that the drag magnitude for flows nearly perpendicular to the major axis of the orography should be somewhere in between those calculated for the 2D and axisymmetric limit cases. There is no reason to doubt that this may also apply to the trapped lee wave drag.

Objections might also be raised about the fact that the present calculations are linear, and therefore formally valid only for infinitesimally small orography. The linear approach is nevertheless useful as a first step in the systematic study of the physical processes addressed here, and as a working assumption almost inevitable in the formulation of parametrizations, since it is the only way that simple analytical expressions for the drag may be obtained. Preliminary results (not shown) suggest that the drag behaviour described here does not change very much for weakly nonlinear flow (e.g. a dimensionless mountain height of $l_{1} h_{0}=0.5$ ), but more definite conclusions about the impacts of finite orography amplitude would require a more comprehensive exploration of the role of nonlinearity. Finally, there are the decisive questions of how to include the important effect of the boundary layer on the wind profile, of whether the idealized stratification profiles considered in the present calculations may be easily fitted to real conditions, and of how to estimate the key input parameters of the drag models developed here in practice. Those are left as topics for future investigations.

\section{Acknowledgement}

M.A.C.T. acknowledges funding from the European Commission, under Marie Curie Career Integration Grant GLIMFLO, contract PCIG13-GA-2013-618016.

\section{Appendix. Setup of numerical simulations}

All of the numerical simulations shown in this paper used the NH3D non-hydrostatic mesoscale numerical model. The basic characteristics of this model are well documented in Miranda (1990) and Miranda and James (1992), and the model has been used extensively in the simulation of mountain waves (Teixeira et al. 2008; Teixeira and Miranda 2009). All model runs were inviscid, 
adiabatic and without rotation. The computational parameters of the runs, including aspects such as sponges, were carefully adjusted through an extensive programme of sensitivity tests (not shown) aimed at achieving maximum accuracy for all considered conditions.

In the runs for the atmosphere of Scorer (1949), the following values for the atmospheric parameters were used: $U=10 \mathrm{~m} \mathrm{~s}^{-1}$, the Brunt-Väisälä frequency in the lower and upper layers were $N_{1}=0.02 \mathrm{~s}^{-1}$ and $N_{2}=0.004 \mathrm{~s}^{-1}$, respectively, so that $l_{1}=$ $2 \times 10^{-3} \mathrm{~m}^{-1}$ and $l_{2}=4 \times 10^{-4} \mathrm{~m}^{-1}$, and $l_{2} / l_{1}=0.2$. The assumed orography parameters were $h_{0}=10 \mathrm{~m}, a=5 \mathrm{~km}, 2.5 \mathrm{~km}$ and $1 \mathrm{~km}$, so that $l_{1} h_{0}=0.02$ and $l_{1} a=10,5$ and $2 . H$ varied between 0 and $5 \mathrm{~km}$, allowing $l_{1} H$ to vary between 0 and 10 . A domain of $512 \times 256$ grid points in the horizontal by 200 equally-spaced sigma levels in the vertical (up to $z \approx 17.5 \mathrm{~km}$ ) was considered, with horizontal spacing proportional to $a$. Hence, for $a=5,2.5,1 \mathrm{~km}$, the grid spacings were $\Delta x=500,250,100 \mathrm{~m}$, respectively, allowing 10 grid points per $a$. The domain had a similar extent (measured from the orography in the middle) in the upstream and lateral boundaries, but extended for a distance 3 times larger in the downstream direction, to allow the trapped lee waves to develop. Raymond-Kuo-type open lateral boundary conditions were used at all lateral boundaries, which effectively prevented wave reflections, but lateral sponges were also applied in the outer 10 grid points at the inflow boundary and in the outer 20 grid points at the outflow boundary, to prevent the imposed incoming flow from drifting in time. A vertical sponge was applied from the top of the domain down to $z \approx 10 \mathrm{~km}$ to prevent spurious vertical wave reflections. Both the duration of each simulation and the time step were proportional to $a / U$, being of $25000 \mathrm{~s}, 12500 \mathrm{~s}$ and $5000 \mathrm{~s}$ and $\Delta t=0.5 \mathrm{~s}, 0.25 \mathrm{~s}$, and $0.1 \mathrm{~s}$ for $l_{1} a=10,5,2$, respectively. The drag was averaged over the final $25 \%$ of the run-time, but it was always ensured that it did not oscillate too much at this stage.

The model runs for the atmosphere of Vosper (2004) used the following values for the atmospheric parameters: $U=10 \mathrm{~ms}^{-1}$, $N_{1}=0, N_{2}=0.01 \mathrm{~s}^{-1}$, yielding $l_{1}=0$ and $l_{2}=1 \times 10^{-3} \mathrm{~m}^{-1}$. The orography parameters were $h_{0}=10 \mathrm{~m}$ and $a=5,2,1,0.5 \mathrm{~km}$. This corresponds to $l_{2} h_{0}=0.01$ and $l_{2} a=5,2,1$ and 0.5 . $H$ was set as $500 \mathrm{~m}$, allowing $l_{2} H$ to take the value of 0.5 used in all simulations. The jump in potential temperature at $z=H$ was varied between $0.65 \mathrm{~K}$ and $65 \mathrm{~K}$ to allow the reduced gravity to vary between $0.0225 \mathrm{~m} \mathrm{~s}^{-2}$ and $2.25 \mathrm{~m} \mathrm{~s}^{-2}$, and consequently the Froude number to vary between 0.3 and 3 . A domain of $256 \times 128$ grid points in the horizontal by 200 equally-spaced sigma levels in the vertical was considered, with horizontal spacing proportional to $a$. Hence, for $a=5,2,1$ and $0.5 \mathrm{~km}$, the grid spacings were $\Delta x=1000,400,200,100 \mathrm{~m}$, respectively, allowing 5 grid points per $a$. The relative domain extent in the upstream, downstream and lateral directions was as for the atmosphere of Scorer (1949), to allow the trapped lee waves to develop. Lateral sponges had the same specifications as for the atmosphere of Scorer (1949) in terms of gridpoints, but extended further into the domain because of the larger grid spacing. Lateral boundary conditions and characteristics of the vertical sponge were similar. The duration and time step of each simulation were calculated in a similar way, yielding durations of $50000 \mathrm{~s}, 20000 \mathrm{~s}, 10000 \mathrm{~s}$ and $5000 \mathrm{~s}$, and $\Delta t=1 \mathrm{~s}, 0.4 \mathrm{~s}, 0.2 \mathrm{~s}$ and $0.1 \mathrm{~s}$, for $l_{2} a=5,2,1,0.5$, respectively. The drag was evaluated and averaged in a similar way.

\section{References}

Bretherton, F.P. 1969. Momentum transport by gravity waves. Q. J. R. Meteorol. Soc. 95, 213-243.

Broutman, D., Rottman, J.W. and Eckermann, S.D. 2003. A simplified Fourier method for nonhydrostatic mountain waves. J. Atmos Sci. 60, 2686-2696.

Cassano, J. 2014. Observations of atmospheric boundary layer temperature profiles with a small unmanned aerial vehicle. Antarctic Sci. 26, 205-213.

Crapper, G.D. 1959. A three-dimensional solution for waves in the lee of mountains. J. Fluid Mech. 6, 51-76.

Doyle, J.D. and Durran, D.R. 2007. Rotor and subrotor dynamics in the lee of three-dimensional terrain. J. Atmos. Sci. 64, 4202-4221.

Esler, J.G., Rump, O.J. and Johnson, E.R. 2007. Non-dispersive and weakly-dispersive single-layer flow over an axisymmetric obstacle: the equivalent aerofoil formulation. J. Fluid Mech. 574, 209-237. 
Gill, A.E. 1982. Atmosphere-Ocean Dynamics, Academic Press, pp. 662.

Gregory, D., Shutts, G.J. and Mitchell, J.R. 1998. A new gravity-wave-drag scheme incorporating anisotropic orography and low-level wave breaking. Impact upon the climate of the UK Meteorological Office Unified Model. Q. J. R. Meteorol. Soc. 124, 463-493.

Héreil, P. and Stein, J. 1999. Momentum budgets over idealized orography with a non-hydrostatic anelastic model. II: Threedimensional flows. Q. J. R. Meteorol. Soc. 125, 2053-2073.

Jiang, Q. and Smith, R.B. 2000. V-waves, bow shocks, and wakes in supercritical hydrostatic flow. J. Fluid Mech. 406, $27-53$.

Lacaze, L., Paci, A., Cid. E., Cazin, S., Eiff, O., Esler, J.G. and Johnson, E.R. 2013. Wave patterns generated by an axisymmetric obstacle in a two-layer flow. Exp. Fluids 54, 1618.

Lighthill, J. 1978. Waves in Fluids, Cambridge University Press, 504 pp.

Lott, F. and Miller, M.J. 1997. A new subgrid-scale orographic drag parametrization: its formulation and testing. Q. J. R. Meteorol. Soc. 123, 101-127.

Marthinsen, T. 1980. Three-dimensional lee waves. Q. J. R. Meteorol. Soc. 106, 569-580.

McPhee, M.G. and Kantha, L.H. 1989. Generation of internal waves by sea ice. J. Geophys. Res. 94, 3287-3302.

Miranda, P.M.A. 1990. Gravity waves and wave drag in flow past three-dimensional isolated mountains. PhD thesis, University of Reading, Reading, UK, 191 pp.

Miranda, P.M.A. and James, I.N. 1992. Nonlinear 3-dimensional effects on gravity-wave drag - splitting flow and breaking waves. $Q$. J. R. Meteorol. Soc. 118, 1057-1081.

Peltier, W.R. and Clark, T.L. 1983. Nonlinear mountain waves in two and three spatial dimensions. Q. J. R. Meteorol. Soc. 109, 527548.

Phillips, D.S. 1984. Analytical surface pressure and drag for linear hydrostatic flow over three-dimensional elliptical mountains. $J$. Atmos. Sci. 41, 1073-1084.

Sachsperger, J., Serafin, S. and Grubisic, V. 2015. Lee waves on the boundary-layer inversion and their dependence on free-atmospheric stability. Front. Earth Sci. 3, 70.

Sachsperger, J., Serafin, S. and Grubisic, V. 2016. Dynamics of rotor formation in uniformly stratified two-dimensional flow over a mountain. Q. J. R. Meteorol. Soc. 142, 1201-1212.

Sawyer, J.S. 1962. Gravity waves in the atmosphere as a three-dimensional problem. Q. J. R. Meteorol. Soc. 88, $412-425$.

Scorer, R.S. 1949. Theory of waves in the lee of mountains. Q. J. R. Meteorol. Soc. 75, 41-56.

Scorer, R.S. 1956. Airflow over an isolated hill. Q. J. R. Meteorol. Soc. 82, 75-81.

Scorer, R.S. and Wilkinson, M. 1956. Waves in the lee of an isolated hill. Q. J. R. Meteorol. Soc. 82, 419-427.

Sharman, R.D. and Wurtele, M.G. 1983. Ship waves and lee waves. J. Atmos. Sci. 40, 396-427.

Sharman, R.D. and Wurtele, M.G. 2004. Three-dimensional structure of forced gravity waves and lee waves. J. Atmos. Sci. 61, 664-681.

Simard, A. and Peltier, W.R. 1982. Ship waves in the lee of isolated topography. J. Atmos. Sci. 39, 587-609.

Smith, R.B. 1976. The generation of lee waves by Blue Ridge. J. Atmos. Sci. 33, 507-519.

Steeneveld, G.J., Holtslag, A.A.M., Nappo, C.J., van de Wiel, B.J.H. and Mahrt, L. 2008. Exploring the possible role of small-scale terrain drag on stable boundary layers over land. J. Appl. Meteorol. Climatol. 47, 2518-2530.

Stensrud, D.J. 2009. Parametrization Schemes: Keys to Understanding Numerical Weather Prediction Models, Cambridge University Press, pp. 459.

Teixeira, M.A.C. 2014. The physics of orographic gravity wave drag. Front. Phys. - Atmos. Sci. 2, 43. 
Teixeira, M.A.C. 2017. Diagnosing lee wave rotor onset using a linear model including a boundary layer. Atmosphere $\mathbf{8}, 5$.

Teixeira, M.A.C., Argaín, J.L. and Miranda, P.M.A. 2013. Drag produced by trapped lee waves and propagating mountain waves in a two-layer atmosphere. Q. J. R. Meteorol. Soc. 139, 964-981.

Teixeira, M.A.C., Argaín, J.L. and Miranda, P.M.A. 2013. Orographic drag associated with lee waves trapped at an inversion. J. Atmos. Sci. 70, 2930-2947.

Teixeira, M.A.C. and Miranda, P.M.A. 2009. On the momentum fluxes associated with mountain waves in directionally sheared flows. J. Atmos. Sci. 66, 3419-3433.

Teixeira, M.A.C., Miranda, P.M.A. and Argaín, J.L. 2008. Mountain waves in two-layer sheared flows: critical level effects, wave reflection, and drag enhancement. J. Atmos. Sci. 65, 1912-1926.

Teixeira, M.A.C., Paci, A. and Belleudy, A. 2017. Drag produced by waves trapped at a density interface in non-hydrostatic flow over an axisymmetric hill. J. Atmos. Sci. 74, 1839-1857.

Teixeira, M.A.C. and Yu, C.-L. 2014. The gravity wave momentum flux in hydrostatic flow with directional shear over elliptical mountains. Eur. J. Mech. B - Fluids 47, 16-31.

Tsiringakis, A., Steeneveld, G.J. and Holtslag, A.A.M. 2017. Small-scale orographic gravity wave drag in stable boundary layers and its impact on synoptic systems and near-surface meteorology. Q. J. R. Meteorol. Soc. 143, 1504-1516.

Vosper, S.B. 2004. Inversion effects on mountain lee waves. Q. J. R. Meteorol. Soc. 130, 1723-1748.

Wurtele, M.G., Sharman, R.D. and Datta, A. 1996. Atmospheric lee waves. Ann. Rev. Fluid Mech. 28, 429-476.

Yu, C.L., Teixeira, M.A.C. 2015. Impact of non-hydrostatic effects and trapped lee waves on mountain-wave drag in directionally sheared flows. Q. J. R. Meterol. Soc. 141, 1572-1585. 